TRANSACTIONS OF THE

AMERICAN MATHEMATICAL SOCIETY

Volume 302, Number 2, August 1987

\title{
VMO, ESV, AND TOEPLITZ OPERATORS \\ ON THE BERGMAN SPACE
}

\author{
KEHE ZHU
}

\begin{abstract}
This paper studies the largest $C^{*}$-subalgebra $Q$ of $L^{\infty}(\mathbf{D})$ such that the Toeplitz operators $T_{f}$ on the Bergman space $L_{a}^{2}(\mathrm{D})$ with symbols $f$ in $Q$ have a symbol calculus modulo the compact operators. $Q$ is characterized by a condition of vanishing mean oscillation near the boundary. I also give several other necessary and sufficient conditions for a bounded function to be in $Q$. After decomposing $Q$ in a "nice" way, I study the Fredholm theory of Toeplitz operators with symbols in $Q$. The essential spectrum of $T_{f}(f \in Q)$ is shown to be connected and computable in terms of the Stone-Cech compactification of D. The results in this article partially answer a question posed in $[\mathbf{3}]$ and give several new necessary and sufficient conditions for a bounded analytic function on the open unit disc to be in the little Bloch space $B_{0}$.
\end{abstract}

1. Introduction. Let $\mathbf{D}$ be the open unit disc in the complex plane $C$. Consider the Bergman space $L_{a}^{2}(\mathbf{D})$ of analytic functions in $L^{2}(\mathbf{D}, d A)$, where $d A=\frac{1}{\pi} r d r d \theta$ is the normalized area measure on $\mathbf{D}$. For any function $f$ in $L^{\infty}(\mathbf{D}, d A)$, the Toeplitz operator $T_{f}: L_{a}^{2}(\mathbf{D}) \rightarrow L_{a}^{2}(\mathbf{D})$ and the Hankel operator $H_{f}: L_{a}^{2}(\mathbf{D}) \rightarrow L^{2}(\mathbf{D})$ are defined by

$$
T_{f} g=P(f g), \quad H_{f} g=(I-P)(f g), \quad g \in L_{a}^{2}(\mathbf{D}),
$$

where $P: L^{2}(\mathbf{D}) \rightarrow L_{a}^{2}(\mathbf{D})$ is the orthogonal projection. It is well known that Toeplitz operators and Hankel operators are related by

$$
T_{|f|^{2}}-T_{\bar{f}} T_{f}=H_{f}^{*} H_{f}
$$

In [3], Sheldon Axler raised the question of characterizing the functions $f \in$ $L^{\infty}(\mathbf{D})$ such that $H_{f}$ is compact. This is equivalent to characterizing functions $f \in L^{\infty}(\mathbf{D})$ such that the semi-self-commutator $T_{|f|^{2}}-T_{\bar{f}} T_{f}$ is compact. Axler answered a special case of this problem in [1]. He proved that for any analytic function $f$ on $\mathbf{D}, H_{\bar{f}}$ is compact if and only if $f \in B_{0}$, the "little Bloch" space.

Recall that for Toeplitz operators $T_{f}$ and Hankel operators $H_{f}\left(f \in L^{\infty}\left(S^{1}\right)\right)$ on the Hardy space $H^{2}$ of the unit circle $S^{1}$, it is well known [15] that $H_{f}$ is compact if and only if $f \in C\left(S^{1}\right)+H^{\infty} ; H_{f}$ and $H_{\bar{f}}$ are compact if and only if $f \in \operatorname{VMO}\left(S^{1}\right)$ $[\mathbf{2 2}, \mathbf{2 3}]$. For Toeplitz operators $T_{f}$ and Hankel operators $H_{f}\left(f \in L^{\infty}\left(C^{n}\right)\right)$ on the Bergman space $L_{a}^{2}\left(C^{n}, d \mu\right)$ of $C^{n}$ with the Gaussian measure $d \mu, \mathrm{L}$. A. Coburn and C. A. Berger in [7] proved that $H_{f}$ is compact if and only if $H_{f}$ and $H_{\bar{f}}$ are compact if and only if $f=f_{1}+f_{2}$ with $f_{1} \in \operatorname{ESV}\left(C^{n}\right)$ and $T_{\left|f_{2}\right|}$ compact.

Received by the editors June 18, 1986.

1980 Mathematics Subject Classification (1985 Revision). Primary 47B35, 30H05; Secondary 32M99, 47G05.

Research partially supported by a grant from the N.S.F.

(C) 1987 American Mathematical Society $0002-9947 / 87 \$ 1.00+\$ .25$ per page 
In this paper, we introduce a new space $\mathrm{VMO}_{\partial}(\mathbf{D})$ of integrable functions on $\mathbf{D}$ and use it to characterize the functions $f \in L^{\infty}(\mathbf{D})$ such that $H_{f}$ and $H_{\bar{f}}$ are compact. $\mathrm{VMO}_{\partial}(\mathbf{D})$, roughly speaking, is the space of integrable functions on $\mathbf{D}$ with vanishing mean oscillation near the boundary of $\mathbf{D}$. The usual area VMO [10] fails to work in this situation because a Toeplitz operator $T_{f}$ on $L_{a}^{2}(\mathbf{D})$, up to a compact perturbation, only depends on the behavior of $f$ near the boundary of $\mathbf{D}$ $[\mathbf{2}, \mathbf{6}, \mathbf{9}, \mathbf{1 6}]$. The (mean) oscillation of $f$ inside $\mathbf{D}$ does not affect $T_{f}$ in the Calkin algebra.

In $\S \S 3$ and 4, we study the space $\mathrm{VMO}_{\partial}(\mathbf{D})$ and one of its important subspaces, $\operatorname{ESV}(\mathbf{D})$. Several equivalent conditions for a function to be in $\mathrm{VMO}_{\partial}(\mathbf{D})$ or $\operatorname{ESV}(\mathbf{D})$ are proved. We also prove that for $f \in H^{\infty}(\mathbf{D}), f \in \mathrm{VMO}_{\partial}(\mathbf{D})$ if and only if $f \in \operatorname{ESV}(\mathbf{D})$ if and only if $f \in B_{0}$. The so-called Berezin symbol [7, 5] serves as a basic tool to study $\mathrm{VMO}_{\partial}(\mathbf{D})$ and Toeplitz operators. Some basic properties of Berezin symbol are first established in $\S 2$. $\S 5$ is devoted to the proof of the main theorem: For $f \in L^{\infty}(\mathbf{D}), H_{f}$ and $H_{\bar{f}}$ are compact if and only if $f$ is in $\operatorname{VMO}_{\partial}(\mathbf{D})$. Notice that this theorem also solves the "symbol calculus" problem of finding the largest $C^{*}$-subalgebra $Q$ of $L^{\infty}(\mathbf{D})$ such that the map $\xi: Q \rightarrow B\left(L_{a}^{2}(\mathbf{D})\right) / K$ defined by $\xi(f)=T_{f}+K$ is a $C^{*}$-algebra homomorphism, where $K$ is the compact ideal of the full algebra $B\left(L_{a}^{2}(\mathbf{D})\right)$ of bounded linear operators on $L_{a}^{2}(\mathbf{D}) . \quad B\left(L_{a}^{2}(\mathbf{D})\right) / K$ is the Calkin algebra. The theorem simply says that $Q=L^{\infty}(\mathbf{D}) \cap \mathrm{VMO}_{\partial}(\mathbf{D})$. In $\S 6$ we discuss the Fredholm theory of Toeplitz operators with symbols in $Q$. The conformal invariance of $\mathrm{VMO}_{\partial}$ is discussed in $\S 7$. $\S 8$ concludes the paper with some open problems and possible generalizations.

2. The Berezin symbol of Toeplitz operators. Recall that $L_{a}^{2}(\mathbf{D})$ has reproducing kernel

$$
K(z, \bar{w})=1 /(1-z \bar{w})^{2} .
$$

For any $w \in \mathbf{D}$, we can define a unit vector $k_{w}$ in $L_{a}^{2}(\mathbf{D})$ by

$$
k_{w}(z)=\frac{K(z, \bar{w})}{\sqrt{K(w, \bar{w})}}=\frac{1-|w|^{2}}{(1-z \bar{w})^{2}}, \quad z \in \mathbf{D} .
$$

The $k_{w}$ 's are called the normalized reproducing kernels.

Now given any bounded linear operator $S$ on $L_{a}^{2}(\mathbf{D})$, we define a bounded continuous function $\tilde{S}$ on $D[\mathbf{5}]$ by

$$
\tilde{S}(z)=\left\langle S k_{z}, k_{z}\right\rangle, \quad z \in \mathbf{D} .
$$

$\tilde{S}$ is called the Berezin symbol of $S$. For any function $f \in L^{\infty}(\mathbf{D}, d A)$, we define $\tilde{f}=\tilde{T}_{f}$, so that

$$
\tilde{f}(z)=\left\langle T_{f} k_{z}, k_{z}\right\rangle=\left\langle f k_{z}, k_{z}\right\rangle=\int_{\mathbf{D}} f(w)\left|k_{z}(w)\right|^{2} d A(w) .
$$

We also call $\tilde{f}$ the Berezin symbol of $f$. Notice that

$$
|\tilde{f}(z)|=\left|\left\langle T_{f} k_{z}, k_{z}\right\rangle\right| \leq\left\|T_{f} k_{z}\right\|\left\|k_{z}\right\| \leq\left\|T_{f}\right\| \leq\|f\|_{\infty}
$$

so $\|\tilde{f}\|_{\infty} \leq\|f\|_{\infty}$. The map $f \mapsto \tilde{f}: L^{\infty}(\mathbf{D}, d A) \rightarrow L^{\infty}(\mathbf{D}, d A)$ is linear, contractive (hence continuous), and order-preserving. It is easy to see that $\tilde{\bar{f}}=\overline{\tilde{f}}$ for any 
$f \in L^{\infty}(\mathbf{D})$. Actually, we have $\tilde{S}^{*}=\overline{\tilde{S}}$ for any bounded linear operator $S$ on $L_{a}^{2}(\mathrm{D})$.

Let $H^{\infty}(\mathbf{D})$ denote the Banach algebra of bounded holomorphic functions on $\mathbf{D}$. We have

Proposition 1. For any $f \in H^{\infty}(\mathbf{D})$ and $z \in \mathbf{D}, T_{\bar{f}} k_{z}=\bar{f}(z) k_{z}$.

PROOF. First recall the reproducing property of $K(z, \bar{w})$ :

$$
f(z)=\int_{\mathbf{D}} K(z, \bar{w}) f(w) d A(w)
$$

for any $f \in L_{a}^{2}(\mathbf{D})$ and $z \in \mathbf{D}$. The Toeplitz operator $T_{f}$ is an integral operator:

$$
\left(T_{f} g\right)(z)=\int_{\mathbf{D}} K(z, \bar{w}) f(w) g(w) d A(w)
$$

for any $f \in L^{\infty}(\mathbf{D})$ and $g \in L_{a}^{2}(\mathbf{D})$. Now if $f \in H^{\infty}(\mathbf{D})$, we have

$$
\begin{aligned}
\left(T_{\bar{f}} K(\cdot, \bar{z})\right)(w) & =\int_{\mathbf{D}} K(w, \bar{u}) K(u, \bar{z}) \bar{f}(u) d A(u) \\
& =\overline{\int_{\mathbf{D}} \overline{K(w, \bar{u})} \overline{K(u, \bar{z})} f(u) d A(u)} \\
& =\overline{\int_{\mathbf{D}} K(u, \bar{w}) K(z, \bar{u}) f(u) d A(u)} \\
& =\overline{K(z, \bar{w}) f(z)}=\bar{f}(z) K(w, \bar{z}),
\end{aligned}
$$

so

$$
T_{\bar{f}} K(\cdot, \bar{z})=\bar{f}(z) K(\cdot, \bar{z}) .
$$

Dividing both sides by $\sqrt{K(z, \bar{z})}$, we get $T_{\bar{f}} k_{z}=\bar{f}(z) k_{z}$.

Proposition 2. For any $f \in H^{\infty}(\mathbf{D})+\overline{H^{\infty}(\mathbf{D})}, \tilde{f}=f$.

PROOF. Since the map $f \mapsto \tilde{f}$ is linear and conjugation-preserving, it suffices to prove the result for $f \in \overline{H^{\infty}(\mathbf{D})}$. But in this case, we have $T_{f} k_{z}=f(z) k_{z}$ by Proposition 1. Thus

$$
\tilde{f}(z)=\left\langle T_{f} k_{z}, k_{z}\right\rangle=\left\langle f(z) k_{z}, k_{z}\right\rangle=f(z)\left\langle k_{z}, k_{z}\right\rangle=f(z) .
$$

PROPOSITION 3. For any $f \in L^{\infty}(\mathbf{D})$, the following are equivalent:

(1) $\lim _{|z| \rightarrow 1^{-}}(\widetilde{f g}(z)-\tilde{f}(z) \tilde{g}(z))=0$ for all $g \in L^{\infty}(\mathbf{D})$;

(2) $\lim _{|z| \rightarrow 1^{-}}\left(\widetilde{|f|^{2}}(z)-|\tilde{f}(z)|^{2}\right)=0$.

PROOF. First it is easy to establish the following two identities:

$$
\begin{aligned}
& \widetilde{|f|^{2}}(z)-|\tilde{f}(z)|^{2}=\frac{1}{2} \int_{\mathbf{D}} \int_{\mathbf{D}}|f(w)-f(u)|^{2}\left|k_{z}(w)\right|^{2}\left|k_{z}(u)\right|^{2} d A(w) d A(u) ; \\
& \widetilde{f g}(z)-\tilde{f}(z) \tilde{g}(z) \\
& \quad=\frac{1}{2} \int_{\mathbf{D}} \int_{\mathbf{D}}(f(u)-f(w))(g(u)-g(w))\left|k_{z}(u)\right|^{2}\left|k_{z}(w)\right|^{2} d A(w) d A(u) .
\end{aligned}
$$

Then the Cauchy-Schwarz inequality gives

$$
\left.|\widetilde{f g}(z)-\tilde{f}(z) \tilde{g}(z)|^{2} \leq\left(\widetilde{|f|^{2}}(z)-|\tilde{f}(z)|^{2}\right) \widetilde{\left(|g|^{2}\right.}(z)-|\tilde{g}(z)|^{2}\right)
$$

Now the desired result follows easily from this inequality. 
COROLLARY

$$
\tilde{Q}=\left\{f \in L^{\infty}(\mathbf{D}) \mid \lim _{|z| \rightarrow 1^{-}}\left(\widetilde{\left.f f\right|^{2}}(z)-|\tilde{f}(z)|^{2}\right)=0\right\}
$$

is a $C^{*}$-subalgebra of $L^{\infty}(\mathbf{D})$.

Proof. Let $f_{1}, f_{2} \in \tilde{Q}$. Then for any $g \in L^{\infty}(\mathbf{D})$, we have

$$
\widetilde{f_{i} g}(z)-\tilde{f_{i}}(z) \tilde{g}(z) \rightarrow 0 \quad \text { as }|z| \rightarrow 1^{-}, i=1,2 .
$$

So

$$
\begin{aligned}
\widetilde{\left(f_{1}+f_{2}\right) g}(z)- & \left(\widetilde{f_{1}+f_{2}}\right)(z) \tilde{g}(z) \\
& =\widetilde{f_{1} g}(z)-\tilde{f}_{1}(z) \tilde{g}(z)+\widetilde{f_{2} g}(z)-\tilde{f}_{2}(z) \tilde{g}(z) \rightarrow 0
\end{aligned}
$$

as $|z| \rightarrow 1^{-}$, and

$$
\begin{aligned}
\left(\widetilde{\left.f_{1} f_{2}\right) g}(z)-\right. & \widetilde{f_{1} f_{2}}(z) \tilde{g}(z) \\
= & \left.\widetilde{f_{1}\left(f_{2} g\right)}(z)-\tilde{f}_{1}(z) \widetilde{f_{2} g}(z)+\tilde{f}_{1}(z) \widetilde{\left(f_{2} g\right.}(z)-\tilde{f}_{2}(z) \tilde{g}(z)\right) \\
& -\left(\widetilde{f_{1} f_{2}}(z)-\tilde{f}_{1}(z) \tilde{f}_{2}(z)\right) \tilde{g}(z)-, 0 \quad \text { as }|z| \rightarrow 1^{-},
\end{aligned}
$$

thus $f_{1}+f_{2}$ and $f_{1} f_{2}$ are in $\tilde{Q}$ by the previous proposition. $\tilde{Q}$ is obviously selfadjoint and closed under scalar multiplication. $\tilde{Q}$ is norm-closed since $f \mapsto \tilde{f}$ is continuous. Therefore, $\tilde{Q}$ is a $C^{*}$-subalgebra of $L^{\infty}(\mathbf{D})$.

Before going on, we have some remarks: then

(1) $k_{z} \rightarrow 0$ weakly in $L_{a}^{2}(\mathbf{D})$ as $|z| \rightarrow 1^{-}$, so if $S$ is a compact operator on $L_{a}^{2}(\mathbf{D})$,

$$
\tilde{S}(z)=\left\langle S k_{z}, k_{z}\right\rangle \rightarrow 0 \quad \text { as }|z| \rightarrow 1^{-} .
$$

(2) If $f$ is a polynomial in $z$, then it is well known that $T_{|f|^{2}}-T_{f} T_{\bar{f}}$ is compact $[9]$, so

$$
\widetilde{|f|^{2}}(z)-|\tilde{f}(z)|^{2}=\widetilde{|f|^{2}}(z)-|f(z)|^{2}=\left\langle\left(T_{|f|^{2}}-T_{f} T_{\bar{f}}\right) k_{z}, k_{z}\right\rangle \rightarrow 0 \quad \text { as }|z| \rightarrow 1^{-} \text {. }
$$

Propositions 1 and 2 are used here. Thus $\tilde{Q}$ contains all the polynomials. By the Stone-Weierstrass theorem, $\tilde{Q}$ contains $C(\overline{\mathbf{D}})$, the algebra of all continuous complex-valued functions on the closed disc $\overline{\mathbf{D}}$.

(3) Propositions 1-3 extend to general domains in $C^{n}$ without change of proofs. However, for an arbitrary domain $\Omega$ in $C^{n}$, one does not have $k_{z} \rightarrow 0$ weakly as $z$ goes to the boundary of $\Omega$.

Let $\tilde{B}=\left\{f \in L^{\infty}(\mathbf{D}) \mid \tilde{f}(z) \rightarrow 0\right.$ as $\left.|z| \rightarrow 1^{-}\right\}$.

Proposition 4. $\tilde{Q} \cap \tilde{B}$ is a closed selfadjoint ideal of $\tilde{Q}$, and the following conditions are all equivalent:

(1) $f \in \tilde{Q} \cap \tilde{B}$

(2) $|f| \in \tilde{B}$;

(3) $|f|^{2} \in \tilde{B}$.

PROOF. If $f \in \tilde{Q} \cap \tilde{B}$ and $g \in \tilde{Q}$, then $\widetilde{f g}(z)=\widetilde{(f g}(z)-\tilde{f}(z) \tilde{g}(z))+\tilde{f}(z) \tilde{g}(z) \rightarrow 0$ as $|z| \rightarrow 1$, so $f g \in \tilde{Q} \cap \tilde{B}$. Thus $\tilde{Q} \cap \tilde{B}$ is an ideal in $\tilde{Q}$. The selfadjointness and closedness (in the sup-norm topology) of $\tilde{Q} \cap \tilde{B}$ are trivial. 
Next we prove that (1)-(3) are all equivalent.

$(2) \Leftrightarrow(3)$ follows from the following inequalities:

$$
\begin{aligned}
\widetilde{|f|^{2}}(z) & =\int_{\mathbf{D}}|f(w)|^{2}\left|k_{z}(w)\right|^{2} d A(w) \\
& \leq\|f\|_{\infty} \int_{\mathbf{D}}|f(w)|\left|k_{z}(w)\right|^{2} d A(w)=\|f\|_{\infty} \widetilde{|f|}(z) \\
\widetilde{|f|}(z) & =\int_{\mathbf{D}}|f(w)|\left|k_{z}(w)\right|^{2} d A(w) \\
& \leq \sqrt{\int_{\mathbf{D}}|f(w)|^{2}\left|k_{z}(w)\right|^{2} d A(w)}=\sqrt{\widetilde{|f|^{2}}(z)}
\end{aligned}
$$

$(3) \Rightarrow(1)$. Suppose $|f|^{2} \in \tilde{B}$, i.e. $\widetilde{|f|^{2}}(z) \rightarrow 0$ as $|z| \rightarrow 1^{-}$. Then

$$
0 \leq \widetilde{|f|^{2}}(z)-|\tilde{f}(z)|^{2} \leq \widetilde{|f|^{2}}(z) \rightarrow 0 \quad\left(|z| \rightarrow 1^{-}\right),
$$

so $f \in \tilde{Q}$. But $|\tilde{f}(z)| \leq \widetilde{|f|} \mid(z) \rightarrow 0\left(|z| \rightarrow 1^{-}\right)$, so we have $f \in \tilde{Q} \cap \tilde{B}$.

$(1) \Rightarrow(3)$. If $f \in \tilde{Q} \cap \tilde{B}$, then $\tilde{f}(z) \rightarrow 0$ and $\widetilde{\left.f\right|^{2}}(z)-|\tilde{f}(z)|^{2} \rightarrow 0$ as $|z| \rightarrow 1^{-}$, so $\widetilde{|f|^{2}}(z) \rightarrow 0\left(|z| \rightarrow 1^{-}\right)$, i.e. $|f|^{2} \in \tilde{B}$.

In [7], Coburn and Berger pointed out that for Toeplitz operators on $L_{a}^{2}\left(C^{n}, d \mu\right)$, where $d \mu$ is the so-called Gaussian measure on $C^{n}$, the Berezin symbol $\tilde{f}$ is just the solution of the heat equation on $C^{n}=\mathbf{R}^{2 n}$ at time $t=\frac{1}{2}$ with initial values $f$. We expect that the same thing happens on the unit disc, but no such equation has been found yet.

3. $\mathrm{VMO}_{\partial}(\mathbf{D})$. For any $z \in \mathbf{D}$, let

$$
S_{z}=\{w \in \mathbf{D}|| w|\geq| z|,| \arg w-\arg z|\leq 1-| z \mid\} .
$$

Now we can give the definition of $\mathrm{VMO}_{\partial}(\mathbf{D})$.

DEFINITION. A function $f \in L^{1}(\mathbf{D}, d A)$ is in $\mathrm{VMO}_{\partial}(\mathbf{D})$ if

$$
\lim _{|z| \rightarrow 1^{-}} \frac{1}{\left|S_{z}\right|} \int_{S_{z}}\left|f(w)-\frac{1}{\left|S_{z}\right|} \int_{S_{z}} f(u) d A(u)\right| A(w)=0,
$$

where $\left|S_{z}\right|=(1+|z|)(1-|z|)^{2}$ is the measure of $S_{z}$ and $\mathrm{VMO}_{\partial}$ stands for "vanishing mean oscillation near the boundary".

The main theorem of this section is the equality

$$
\tilde{Q}=L^{\infty}(\mathbf{D}) \cap \mathrm{VMO}_{\partial}(\mathbf{D}) .
$$

LEMMA 1. If $\delta \in(0,1)$ is close enough to 1 , then $|1-z| \leq\left|1-\delta e^{i(1-\delta)}\right|$ for all $z$ in $S_{\delta}$.

Proof. Given $z \in S_{\delta}$, write $z=r e^{i \theta}$. Then

$$
\begin{aligned}
\left|1-\delta e^{i(1-\delta)}\right|^{2}-|1-z|^{2} & =\delta^{2}-2 \delta \cos (1-\delta)-r^{2}+2 r \cos \theta \\
& \geq \delta^{2}-2 \delta \cos (1-\delta)-r^{2}+2 r \cos (1-\delta) \\
& =(\delta-r)(\delta+r)-2(\delta-r) \cos (1-\delta) \\
& =(r-\delta)(2 \cos (1-\delta)-\delta-r) \\
& \geq(r-\delta)(2 \cos (1-\delta)-1-\delta) .
\end{aligned}
$$


For $\delta$ close enough to 1 , we have

$$
\cos (1-\delta) \geq 1-(1-\delta)^{2} / 2,
$$

thus

$$
2 \cos (1-\delta)-1-\delta \geq 2-(1-\delta)^{2}-1-\delta=2 \delta-\delta^{2}-\delta=\delta-\delta^{2}>0 .
$$

This completes the proof of the lemma.

LEMMA 2. If $\delta \in(0,1)$ is very close to 1 , then $\left|1-\delta e^{i(1-\delta)}\right| \leq 2(1-\delta)$.

ProOF. The equality

$$
\left|1-\delta e^{i(1-\delta)}\right|^{2}=1+\delta^{2}-2 \delta \cos (1-\delta)
$$

and L'Hôpital's rule give us the limit

$$
\lim _{\delta \rightarrow 1^{-}} \frac{\left|1-\delta e^{i(1-\delta)}\right|^{2}}{(1-\delta)^{2}}=2 .
$$

So for $\delta$ close enough to 1 , we must have

$$
\left|1-\delta e^{i(1-\delta)}\right|^{2} \leq 4(1-\delta)^{2}
$$

LEMMA 3. For any $\varepsilon>0$, there are $\sigma$ and $\delta_{0}$ in $(0,1)$ such that

$$
\int_{\mathbf{D}-S_{\delta e^{i \theta}}} \frac{\left(1-|z|^{2}\right)^{2}}{|1-\bar{z} w|^{4}} d A(w)<\varepsilon
$$

whenever $z=|z| e^{i \theta} \in \mathbf{D}, 0<1-|z|<\delta_{0}$, and $1-|z|=\sigma(1-\delta)$.

PROOF. Let $r=|z|$. A change of variable gives

$$
\int_{\mathbf{D}-S_{\delta e^{i \theta}}} \frac{\left(1-|z|^{2}\right)^{2}}{|1-\bar{z} w|^{4}} d A(w)=\int_{\mathbf{D}-S_{\delta}} \frac{\left(1-r^{2}\right)^{2}}{|1-r w|^{4}} d A(w)=\frac{1}{\pi} \operatorname{Area} F\left(\mathbf{D}-S_{\delta}\right),
$$

where $F: \mathbf{D} \rightarrow \mathbf{D}$ is the map defined by $F(w)=(r-w) /(1-r w)$.

Notice that we have used the fact that $\left(1-|z|^{2}\right)^{2} /|1-\bar{z} w|^{4}$ is the Jacobian of the map $w \mapsto(z-w) /(1-\bar{z} w)$.

Now suppose that $\sigma$ is any number in $(0,1)$ and $1-r=\sigma(1-\delta)$. We want to estimate $|1-F(w)|$ for all $w$ in $\mathbf{D}-S_{\delta}$. If $w \in \mathbf{D}-S_{\delta}$, then either $|w|<\delta$ or $|w| \geq \delta$ but $|\arg w|>1-\delta$.

Case 1. $|w|<\delta$. In this case, we have

$$
\begin{aligned}
|1-F(w)| & =\left|1-\frac{r-w}{1-r w}\right|=\frac{(1-r)|1+w|}{|1-r w|} \\
& \leq \frac{2(1-r)}{1-r \delta}=\frac{2 \sigma(1-\delta)}{1-\delta(1-\sigma(1-\delta))} \\
& =\frac{2 \sigma(1-\delta)}{(1-\delta)(1+\sigma \delta)}=\frac{2 \sigma}{1+\sigma \delta} \leq 2 \sigma
\end{aligned}
$$

Case 2. $|w| \geq \delta,|\arg w|>1-\delta$. In this case, we have

$$
\begin{aligned}
|1-r w|^{2} & \geq 1+r^{2}|w|^{2}-2 r|w| \cos (1-\delta) \\
& \geq 1+r^{2}|w|^{2}-2 r|w|\left(1-(1-\delta)^{2} / 2+(1-\delta)^{4} / 24\right) \\
& =(1-r|w|)^{2}+r|w|(1-\delta)^{2}-r|w|(1-\delta)^{4} / 12 \\
& \geq(1-r)^{2}+\delta r(1-\delta)^{2}-\frac{1}{12}(1-\delta)^{4} \\
& =(1-\delta)^{2}\left(\sigma^{2}+\delta r-\frac{1}{12}(1-\delta)^{2}\right),
\end{aligned}
$$


thus

$$
\begin{aligned}
|1-F(w)| & =\frac{(1-r)|1+w|}{|1-r w|} \leq \frac{2(1-\delta) \sigma}{|1-r w|} \\
& \leq \frac{2 \sigma(1-\delta)}{(1-\delta) \sqrt{\sigma^{2}+\delta r-\frac{1}{12}(1-\delta)^{2}}}=\frac{2 \sigma}{\sqrt{\sigma^{2}+\delta r-\frac{1}{12}(1-\delta)^{2}}}
\end{aligned}
$$

Since we are only concerned with $\delta$ close to 1 , we may assume $\delta>\frac{1}{2}\left(\Rightarrow r>\delta>\frac{1}{2}\right)$. Thus

which gives $|1-F(w)| \leq 6 \sigma$.

$$
\sigma^{2}+\delta r-\frac{1}{12}(1-\delta)^{2}>\frac{1}{2} \cdot \frac{1}{2}-\frac{1}{12}\left(1-\frac{1}{2}\right)^{2}>\frac{1}{9},
$$

Combining Cases 1 and 2, we have proved that $|1-F(w)| \leq 6 \sigma$ whenever $w \in \mathbf{D}-S_{\delta}, 1-r=\sigma(1-\delta)\left(\delta>\frac{1}{2}\right)$. Therefore, if $\sigma$ is small enough, $F\left(\mathbf{D}-S_{\delta}\right)$ is concentrated around 1 , so Area $F\left(\mathbf{D}-S_{\delta}\right)$ is small. This completes the proof of Lemma 3. [Note: $\delta>\frac{1}{2} \Rightarrow 1-r=\sigma(1-\delta)<\frac{1}{2} \sigma$, so $\delta_{0}$ can be chosen to be $\frac{1}{2} \sigma$.]

LEMMA 4. For $f \in L^{\infty}(\mathbf{D})$, we have

$$
\begin{aligned}
& \frac{1}{\left|S_{z}\right|} \int_{S_{z}}\left|f(w)-\frac{1}{\left|S_{z}\right|} \int_{S_{z}} f(u) d A(u)\right| d A(w) \\
& \leq \sqrt{\frac{1}{\left|S_{z}\right|^{2}} \int_{S_{z}} \int_{S_{z}}|f(w)-f(u)|^{2} d A(w) d A(u)} \\
& \frac{1}{\left|S_{z}\right|^{2}} \int_{S_{z}} \int_{S_{z}}|f(w)-f(u)|^{2} d A(w) d A(u) \\
& \leq 4\|f\|_{\infty} \frac{1}{\left|S_{z}\right|} \int_{S_{z}}\left|f(w)-\frac{1}{\left|S_{z}\right|} \int_{S_{z}} f(u) d A(u)\right| d A(w) .
\end{aligned}
$$

PROOF. (1) follows from the Cauchy-Schwarz inequality, while (2) follows from the following identity:

$$
\begin{aligned}
\frac{1}{\left|S_{z}\right|^{2}} \int_{S_{z}} & \int_{S_{z}}|f(w)-f(u)|^{2} d A(w) d A(u) \\
= & \frac{2}{\left|S_{z}\right|} \int_{S_{z}} \bar{f}(w)\left(f(w)-\frac{1}{\left|S_{z}\right|} \int_{S_{z}} f(u) d A(u)\right) d A(w) \\
& +\frac{2}{\left|S_{z}\right|} \int_{S_{z}} \bar{f}(w) d A(w) \cdot \frac{1}{\left|S_{z}\right|} \int_{S_{z}}\left(f(w)-\frac{1}{\left|S_{z}\right|} \int_{S_{z}} f(u) d A(u)\right) d A(w) .
\end{aligned}
$$

COROllary. For $f \in L^{\infty}(\mathbf{D}), f \in \mathrm{VMO}_{\partial}(\mathbf{D})$ if and only if

$$
\lim _{|z| \rightarrow 1^{-}} \frac{1}{\left|S_{z}\right|^{2}} \int_{S_{z}} \int_{S_{z}}|f(w)-f(u)|^{2} d A(w) d A(u)=0
$$

THEOREM 1. $\tilde{Q}=\mathrm{VMO}_{\partial}(\mathbf{D}) \cap L^{\infty}(\mathbf{D})$.

PROOF. First we prove the inclusion $\tilde{Q} \subset \mathrm{VMO}_{\partial}(\mathbf{D})$. Given $z=|z| e^{i \theta} \in \mathbf{D}$ and $f \in \tilde{Q}$,

$$
\begin{aligned}
\widetilde{|f|^{2}}(z)-|\tilde{f}(z)|^{2} & =\frac{1}{2}\left(1-|z|^{2}\right)^{4} \int_{\mathbf{D}} \int_{\mathbf{D}} \frac{|f(w)-f(u)|^{2}}{|1-\bar{z} w|^{4}|1-\bar{z} u|^{4}} d A(w) d A(u) \\
& \geq \frac{1}{2}\left(1-|z|^{2}\right)^{4} \int_{S_{z}} \int_{S_{z}} \frac{|f(w)-f(u)|^{2}}{|1-\bar{z} w|^{4}|1-\bar{z} u|^{4}} d A(w) d A(u) .
\end{aligned}
$$


For $w, u \in S_{z}$, we have $\bar{z} w, \bar{z} u \in S_{|z|^{2}}$. Thus if $|z|$ is close enough to 1 ,

$$
|1-\bar{z} w| \leq\left.|1-| z\right|^{2} e^{i\left(1-|z|^{2}\right)} \mid \leq 2\left(1-|z|^{2}\right)
$$

by Lemmas 1 and 2 . Similarly, $|1-\bar{z} u| \leq 2\left(1-|z|^{2}\right)$. So

$$
\widetilde{|f|^{2}}(z)-|\tilde{f}(z)|^{2} \geq \frac{1}{2^{9}} \cdot \frac{1}{\left(1-|z|^{2}\right)^{4}} \int_{S_{z}} \int_{S_{z}}|f(w)-f(u)|^{2} d A(w) d A(u) .
$$

Notice that

$$
\left|S_{z}\right|^{2}=(1+|z|)^{2}(1-|z|)^{4} \sim\left(1-|z|^{2}\right)^{4}
$$

So we have

$$
\widetilde{|f|^{2}}(z)-|\tilde{f}(z)|^{2} \rightarrow 0 \Rightarrow \lim _{|z| \rightarrow 1} \frac{1}{\left|S_{z}\right|^{2}} \int_{S_{z}} \int_{S_{z}}|f(w)-f(u)|^{2} d A(w) d A(u)=0
$$

which means $f \in \tilde{Q} \Rightarrow f \in \mathrm{VMO}_{\partial}(\mathbf{D})$ by Lemma 4 .

Next we prove the other inclusion:

$$
\mathrm{VMO}_{\partial}(\mathbf{D}) \cap L^{\infty}(\mathbf{D}) \subset \tilde{Q} .
$$

Given $z=|z| e^{i \theta} \in \mathbf{D}, \delta \in(0,1)$, and $f \in \mathrm{VMO}_{\partial}(\mathbf{D}) \cap L^{\infty}(\mathbf{D})$,

$$
\begin{aligned}
& \widetilde{|f|^{2}}(z)-|\tilde{f}(z)|^{2}=\frac{1}{2} \int_{\mathbf{D}} \int_{\mathbf{D}} \frac{\left(1-|z|^{2}\right)^{4}|f(w)-f(u)|^{2}}{|1-z \bar{w}|^{4}|1-z \bar{u}|^{4}} d A(w) d A(u) \\
& =\frac{1}{2}\left(1-|z|^{2}\right)^{4} \int_{S_{\delta e^{i \theta}}} \int_{S_{\delta e^{i \theta}}} \frac{|f(w)-f(u)|^{2}}{|1-\bar{z} w|^{4}|1-\bar{z} u|^{4}} d A(w) d A(u) \\
& +\frac{1}{2}\left(1-|z|^{2}\right)^{4}\left[\int_{\mathbf{D}-S_{\delta e^{i \theta}}} \int_{\mathbf{D}}+\int_{\mathbf{D}} \int_{\mathbf{D}-S_{\delta e^{i \theta}}}-\int_{\mathbf{D}-S_{\delta e^{i \theta}}} \int_{\mathbf{D}-S_{\delta e^{i \theta}}}\right] \\
& \frac{|f(w)-f(u)|^{2}}{|1-\bar{z} w|^{4}|1-\bar{z} u|^{4}} d A(w) d A(u) \\
& \leq \frac{1}{2}\left(1-|z|^{2}\right)^{4} \int_{S_{\delta e^{i \theta}}} \int_{S_{\delta e^{i \theta}}} \frac{|f(w)-f(u)|^{2}}{(1-|z|)^{4}(1-|z|)^{4}} d A(w) d A(u) \\
& +\frac{1}{2}\left(1-|z|^{2}\right)^{4}\left[\int_{\mathbf{D}} \int_{\mathbf{D}-S_{\delta e^{i \theta}}}+\int_{\mathbf{D}-S_{\delta e^{i \theta}}} \int_{\mathbf{D}}\right] \\
& \frac{4\|f\|_{\infty}^{2}}{|1-\bar{z} w|^{4}|1-\bar{z} u|^{4}} d A(w) d A(u) \\
& =\frac{1}{2}\left(\frac{1+|z|}{1-|z|}\right)^{4} \int_{S_{\delta e^{i \theta}}} \int_{S_{\delta e^{i \theta}}}|f(w)-f(u)|^{2} d A(u) d A(w) \\
& +4\|f\|_{\infty}^{2}\left(1-|z|^{2}\right)^{2} \int_{\mathbf{D}-S_{\delta e^{i \theta}}} \frac{d A(w)}{|1-\bar{z} w|^{4}} \\
& =\frac{(1+|z|)^{4}(1+\delta)^{2}}{2}\left(\frac{1-\delta}{1-|z|}\right)^{4} \frac{1}{\mid S_{\left.\delta e^{i \theta}\right|^{2}}} \\
& \cdot \int_{S_{\delta e^{i \theta}}} \int_{S_{\delta e^{i} \theta}}|f(w)-f(u)|^{2} d A(w) d A(u) \\
& +4\|f\|_{\infty} \int_{\mathbf{D}-S_{\delta e^{i \theta}}} \frac{\left(1-|z|^{2}\right)^{2}}{|1-\bar{z} w|^{4}} d A(w) \text {. }
\end{aligned}
$$


Now given any $\varepsilon>0$, by Lemma 3 , there exist $\sigma \in(0,1)$ and $\delta_{0} \in(0,1)$ such that

$$
\int_{\mathbf{D}-S_{\delta e^{i \theta}}} \frac{\left(1-|z|^{2}\right)^{2}}{|1-\bar{z} w|^{4}} d A(w)<\varepsilon
$$

whenever $0<1-|z|<\delta_{0}, 1-|z|=(1-\delta) \sigma$. Thus

$$
\begin{aligned}
& \widetilde{|f|^{2}}(z)-|\tilde{f}(z)|^{2} \\
& \quad \leq 4\|f\|_{\infty}^{2} \varepsilon+\frac{(1+|z|)^{4}(1+\delta)^{2}}{2 \sigma^{4}} \frac{1}{\mid S_{\left.\delta e^{i \theta}\right|^{2}}} \int_{S_{\delta e^{i \theta}}} \int_{S_{\delta e^{i \theta}}}|f(w)-f(u)|^{2} d A(w) d A(u)
\end{aligned}
$$

whenever $0<1-|z|<\delta_{0}$ and $1-|z|=(1-\delta) \sigma$.

Now using Lemma 4 we get

$$
\varlimsup_{|z| \rightarrow 1^{-}}\left(\widetilde{|f|^{2}}(z)-|\tilde{f}(z)|^{2}\right) \leq 4\|f\|_{\infty}^{2} \varepsilon .
$$

(Note: $|z| \rightarrow 1 \Rightarrow \delta \rightarrow 1, \sigma$ is fixed.) Since $\varepsilon$ is arbitrary, we have

$$
\lim _{|z| \rightarrow 1^{-}}\left(\widetilde{|f|^{2}}(z)-|\tilde{f}(z)|^{2}\right)=0
$$

and so $f \in \tilde{Q}$. This completes the proof of Theorem 1 .

THEOREM 2. For $f \in L^{\infty}(\mathbf{D}, d A), f \in \tilde{Q} \cap \tilde{B}$ iff

$$
\lim _{|z| \rightarrow 1^{-}} \frac{1}{\left|S_{z}\right|} \int_{S_{z}}|f(w)| d A(w)=0
$$

ProOF. Suppose that (1) holds. Consider

$$
\widetilde{|f|}(z)=\left(1-|z|^{2}\right)^{2} \int_{\mathbf{D}} \frac{|f(w)|}{|1-z \bar{w}|^{4}} d A(w), \quad z=|z| e^{i \theta}
$$

Given $\varepsilon>0$, by Lemma 3 there are $\sigma \in(0,1)$ and $\delta_{0} \in(0,1)$ such that

$$
\int_{\mathbf{D}-S_{\delta e^{i \theta}}} \frac{\left(1-|z|^{2}\right)^{2}}{|1-\bar{z} w|^{4}} d A(w)<\varepsilon
$$

whenever $0<1-|z|<\delta_{0}$ and $1-|z|=(1-\delta) \sigma$, so

$$
\begin{aligned}
\widetilde{|f|}(z) & \leq\|f\|_{\infty} \varepsilon+\left(1-|z|^{2}\right)^{2} \int_{S_{\delta e^{i \theta}}} \frac{|f(w)|}{|1-\bar{z} w|^{4}} d A(w) \\
& \leq\|f\|_{\infty} \varepsilon+\frac{\left(1-|z|^{2}\right)^{2}}{(1-|z|)^{4}} \int_{S_{\delta e^{i \theta}}}|f(w)| d A(w)
\end{aligned}
$$

whenever $0<1-|z|<\delta_{0}$ and $1-|z|=(1-\delta) \sigma$. Since

$$
\frac{\left(1-|z|^{2}\right)^{2}}{(1-|z|)^{4}}=\frac{(1+|z|)^{2}}{(1-|z|)^{2}}=\frac{(1+|z|)^{2}}{\sigma^{2}(1-\delta)^{2}} \leq \frac{4}{\sigma^{2}(1-\delta)^{2}}
$$

and $\left|S_{\delta e^{i \theta}}\right|=(1+\delta)(1-\delta)^{2}$, thus

$$
\widetilde{|f|}(z) \leq\|f\|_{\infty} \varepsilon+\frac{4(1+\delta)}{\sigma^{2}\left|S_{\delta e^{i \theta}}\right|} \int_{S_{\delta e^{i \theta}}}|f(w)| d A(w)
$$


whenever $0<1-|z|<\delta_{0}$ and $1-|z|=\sigma(1-\delta)$. Let $|z| \rightarrow 1^{-}$in (2). Then

$$
\varlimsup_{|z| \rightarrow 1^{-}} \widetilde{|f|}(z) \leq\|f\|_{\infty} \varepsilon
$$

Since $\varepsilon$ is arbitrary, we have $|f| \in \tilde{B}$, thus $f \in \tilde{Q} \cap \tilde{B}$.

Conversely, if $f \in \tilde{Q} \cap \tilde{B}$, then $|f| \in \tilde{B}$. But

$$
\begin{aligned}
\widetilde{|f|}(z) & =\left(1-|z|^{2}\right)^{2} \int_{\mathbf{D}} \frac{|f(w)|}{|1-\bar{z} w|^{4}} d A(w) \\
& \geq\left(1-|z|^{2}\right)^{2} \int_{S_{z}} \frac{|f(w)|}{|1-\bar{z} w|^{4}} d A(w),
\end{aligned}
$$

and $|1-\bar{z} w| \leq 2\left(1-|z|^{2}\right)$ for $|z|$ close enough to 1 and $w \in S_{z}$ by Lemmas 1 and 2. So there is $\delta \in(0,1)$ such that

$$
\widetilde{|f|}(z) \geq \frac{1}{2^{4}\left(1-|z|^{2}\right)^{2}} \int_{S_{z}}|f(w)| d A(w)
$$

for all $\delta<|z|<1$. Notice that $\left(1-|z|^{2}\right)^{2}=(1+|z|)\left|S_{z}\right|$. So (3) says that $|\tilde{f}|(z) \rightarrow 0\left(|z| \rightarrow 1^{-}\right)$implies

$$
\frac{1}{\left|S_{z}\right|} \int_{S_{z}}|f(w)| d A(w) \rightarrow 0 \quad\left(|z| \rightarrow 1^{-}\right)
$$

This completes the proof of Theorem 2 .

4. $\operatorname{ESV}(\mathbf{D})$. Let $f$ be in $L^{\infty}(\mathbf{D}, d A)$. We say $f$ is in $\operatorname{ESV}(\mathbf{D})$ if for any $\varepsilon>0$ and $\sigma \in(0,1)$, there is $\delta_{0}>0$ such that $|f(z)-f(w)|<\varepsilon$ whenever $|z|,|w| \in$ $[1-\delta, 1-\sigma \delta], \delta<\delta_{0}$ and $|\arg z-\arg w| \leq \max (1-|z|, 1-|w|)$.

Recall that for $z \in \mathbf{D}$,

$$
S_{z}=\{w \in \mathbf{D}|| w|\geq| z|,| \arg z-\arg w|\leq 1-| z \mid\} .
$$

Then it is clear that $f \in \operatorname{ESV}(\mathrm{D})$ if and only if for any $\varepsilon>0, \sigma \in(0,1)$, there exists $\delta_{0}>0$ such that $|f(z)-f(w)|<\varepsilon$ whenever $w \in S_{z}$ and $|z|,|w| \in[1-\delta, 1-\sigma \delta], \delta<$ $\delta_{0}$.

It is also easy to see that $f \in \operatorname{ESV}(\mathbf{D})$ if and only if for any $\varepsilon>0, \sigma \in$ $(0,1)$ and for any positive number $k$, there exists a positive number $\delta_{0}$ such that $|f(z)-f(w)|<\varepsilon$ whenever $|z|,|w| \in[1-\delta, 1-\sigma \delta], \delta<\delta_{0}$, and $|\arg z-\arg w| \leq$ $k \max (1-|z|, 1-|w|)$. In particular, if

$$
S_{z}^{\prime}=\{w \in \mathbf{D}|| w|\geq| z|,| \arg w-\arg z \mid \leq(1-|z|) / 2\}
$$

then $f \in \operatorname{ESV}(\mathbf{D})$ if and only if for any $\varepsilon>0$, and $\sigma \in(0,1)$, there exists $\delta_{0}>0$ such that $|f(z)-f(w)|<\varepsilon$ whenever $w \in S_{z}^{\prime}$ and $|z|,|w| \in[1-\delta, 1-\sigma \delta], \delta<\delta_{0}$.

The notation ESV is borrowed from [6] and [7], where it stands for "eventually slowly varying". In [22] and [23], Sarason also introduced the concept of ESV in a special case, but used a different notation, namely, SO, standing for "slowly oscillating". ESV is indeed an oscillation condition. It is stronger than the meanoscillation condition as shown in Theorem 5 .

$\operatorname{ESV}(\mathbf{D})$ is a relatively large class of functions in $L^{\infty}(\mathbf{D}, d A)$. It is easy to see that $C(\overline{\mathbf{D}})$ is strictly contained in it. 
Let $f \in L^{\infty}(\mathbf{D})$ and $z \in \mathbf{D}$, and define

$$
\hat{f}(z)=\frac{1}{\left|S_{z}^{\prime}\right|} \int_{S_{z}^{\prime}} f(w) d A(w) .
$$

REMARK. By the corollary to Lemma 4 , it is easy to see that $f \in \tilde{Q}$ if and only if $|\hat{f}|^{2}(z)-|\hat{f}(z)|^{2} \rightarrow 0$ as $|z| \rightarrow 1^{-}$.

THEOREM 3. If $f \in \tilde{Q}=\operatorname{VMO}_{\partial}(\mathbf{D}) \cap L^{\infty}(\mathbf{D})$, then $\hat{f} \in \operatorname{ESV}(\mathbf{D})$.

Proof. Given $\varepsilon>0, \sigma \in(0,1)$, choose $\delta_{0}>0$ such that

$$
\frac{1}{\left|S_{z}\right|^{2}} \int_{S_{z}} \int_{S_{z}}|f(w)-f(u)| d A(w) d A(u)<\frac{\varepsilon \sigma^{2}}{8}
$$

whenever $0<1-|z|<\delta_{0}$.

Now if $1-\delta \leq\left|z_{1}\right| \leq\left|z_{2}\right| \leq 1-\sigma \delta, \delta<\delta_{0}$, and

$$
\left|\arg z_{1}-\arg z_{2}\right| \leq \frac{1}{2} \max \left(1-\left|z_{1}\right|, 1-\left|z_{2}\right|\right)=\frac{1}{2}\left(1-\left|z_{1}\right|\right)
$$

then $S_{z_{2}}^{\prime} \subset S_{z_{1}}$, so we have

$$
\begin{aligned}
\left|\hat{f}\left(z_{1}\right)-\hat{f}\left(z_{2}\right)\right| & =\left|\frac{1}{\left|S_{z_{1}}^{\prime}\right|\left|S_{z_{2}}^{\prime}\right|} \int_{S_{z_{1}}^{\prime}} \int_{S_{z_{2}}^{\prime}}(f(u)-f(w)) d A(u) d A(w)\right| \\
& \leq \frac{1}{\left|S_{z_{1}}^{\prime}\right|\left|S_{z_{2}}^{\prime}\right|} \int_{S_{z_{1}}^{\prime}} \int_{S_{z_{2}}^{\prime}}|f(u)-f(w)| d A(u) d A(w) \\
& \leq \frac{1}{\left|S_{z_{1}}^{\prime}\right|\left|S_{z_{2}}^{\prime}\right|} \int_{S_{z_{1}}} \int_{S_{z_{1}}}|f(u)-f(w)| d A(u) d A(w) \\
& =\frac{4}{\left|S_{z_{1}}\right|\left|S_{z_{2}}^{\prime}\right|} \int_{S_{z_{1}}} \int_{S_{z_{1}}}|f(u)-f(w)| d A(u) d A(w) .
\end{aligned}
$$

But

$$
\frac{\left|S_{z_{1}}\right|}{\left|S_{z_{2}}\right|}=\frac{\left(1+\left|z_{1}\right|\right)\left(1-\left|z_{1}\right|\right)^{2}}{\left(1+\left|z_{2}\right|\right)\left(1-\left|z_{2}\right|\right)^{2}} \leq 2\left(\frac{1-\left|z_{1}\right|}{1-\left|z_{2}\right|}\right)^{2} \leq \frac{2}{\sigma^{2}} .
$$

(Note: $1-\delta \leq\left|z_{1}\right| \leq\left|z_{2}\right| \leq 1-\sigma \delta \Rightarrow\left(1-\left|z_{1}\right|\right) /\left(1-\left|z_{2}\right|\right) \leq 1 / \sigma$.) So we have $\left|\hat{f}\left(z_{1}\right)-\hat{f}\left(z_{2}\right)\right| \leq \frac{8}{\sigma^{2}} \cdot \frac{1}{\left|S_{z_{1}}\right|^{2}} \int_{S_{z_{1}}} \int_{S_{z_{1}}}|f(u)-f(w)| d A(u) d A(w) \leq \frac{8}{\sigma^{2}} \cdot \frac{\sigma^{2} \varepsilon}{8}=\varepsilon$.

Thus $\hat{f}$ is in $\operatorname{ESV}(\mathbf{D})$.

LEMMA 5. If $f \in \operatorname{ESV}(\mathbf{D})$, then

$$
\lim _{|z| \rightarrow 1^{-}} \frac{1}{\left|S_{z}\right|} \int_{S_{z}}|f(z)-f(u)| d A(u)=0 .
$$

Proof. For $0<|z|<\delta<1$, let

$$
\begin{aligned}
& A_{1}=\left\{w \in S_{z}|| w \mid \leq \delta\right\} \\
& A_{2}=\left\{w \in S_{z}|| w \mid \geq \delta\right\}
\end{aligned}
$$


Then $S_{z}=A_{1} \cup A_{2}$ and

$$
\begin{aligned}
& \frac{1}{\left|S_{z}\right|} \int_{S_{z}}|f(z)-f(u)| d A(u) \\
& \quad \leq \frac{1}{\left|A_{1}\right|} \int_{A_{1}}|f(z)-f(u)| d A(u)+\frac{1}{\left|S_{z}\right|} \int_{A_{2}}|f(z)-f(u)| d A(u) .
\end{aligned}
$$

Given any $\varepsilon \in(0,1)$, choose $\delta_{0} \in(0,1)$ such that

$$
\left|f\left(r_{1} e^{i \theta_{1}}\right)-f\left(r_{2} e^{i \theta_{2}}\right)\right|<\varepsilon
$$

whenever $r_{1}, r_{2} \in[1-r, 1-\varepsilon r], r \leq \delta_{0}$, and $\left|\theta_{1}-\theta_{2}\right| \leq \max \left(1-r_{1}, 1-r_{2}\right)$.

Now let $|z|>1-\delta_{0}$ and $\delta=1-\varepsilon(1-|z|)$. Then $|z|<\delta<1$. For any $u \in A_{1}$, $|f(z)-f(u)|<\varepsilon$ by $(*)$. So for each $|z|>1-\delta_{0}$, we have

$$
\begin{aligned}
\frac{1}{\left|S_{z}\right|} \int_{S_{z}}|f(z)-f(u)| d A(u) & \leq \varepsilon+\frac{1}{\left|S_{z}\right|} \int_{A_{2}}|f(z)-f(u)| d A(u) \\
& \leq \varepsilon+2\left|A_{2}\right| /\left|S_{z}\right|\|f\|_{\infty} .
\end{aligned}
$$

Since

$$
\begin{aligned}
& \left|S_{z}\right|=(1+|z|)(1-|z|)^{2} \\
& \left|A_{2}\right|=(1-|z|)\left(1-\delta^{2}\right)=\varepsilon(1-|z|)^{2}(2-\varepsilon(1-|z|)), \\
& \frac{\left|A_{2}\right|}{\left|S_{z}\right|} \leq \frac{2 \varepsilon(1-|z|)^{2}}{(1+|z|)(1-|z|)^{2}} \leq 2 \varepsilon
\end{aligned}
$$

$|z|>1-\delta_{0}$ implies

$$
\frac{1}{\left|S_{z}\right|} \int_{S_{z}}|f(z)-f(u)| d A(u) \leq\left(1+4\|f\|_{\infty}\right) \varepsilon .
$$

This completes the proof of Lemma 5.

THEOREM 4. If $f \in \tilde{Q}=\operatorname{VMO}_{\partial}(\mathbf{D}) \cap L^{\infty}(\mathbf{D})$, then $f-\hat{f} \in \tilde{Q} \cap \tilde{B}$.

ProOF. Let $g=f-\hat{f}$. Then

$$
\begin{aligned}
\frac{1}{\left|S_{z}\right|} \int_{S_{z}}|g(w)| d A(w) \leq & \frac{1}{\left|S_{z}\right|} \int_{S_{z}}|f(w)-\hat{f}(z)| d A(w) \\
& +\frac{1}{\left|S_{z}\right|} \int_{S_{z}}|\hat{f}(z)-\hat{f}(w)| d A(w)
\end{aligned}
$$

The second term goes to 0 as $|z| \rightarrow 1^{-}$by Lemma 5 and Theorem 3 . Next we estimate the first term.

$$
\begin{aligned}
& \frac{1}{\left|S_{z}\right|} \int_{S_{z}}|f(w)-\hat{f}(z)| d A(w) \\
& \quad=\frac{1}{\left|S_{z}\right|} \int_{S_{z}}\left|\frac{1}{\left|S_{z}^{\prime}\right|} \int_{S_{z}^{\prime}}(f(w)-f(u)) d A(u)\right| d A(w) \\
& \quad \leq \frac{1}{\left|S_{z}\right|\left|S_{z}^{\prime}\right|} \int_{S_{z}} \int_{S_{z}^{\prime}}|f(w)-f(u)| d A(u) \\
& \quad \leq \frac{2}{\left|S_{z}\right|^{2}} \int_{S_{z}} \int_{S_{z}}|f(w)-f(u)| d A(w) d A(u)
\end{aligned}
$$

this goes to 0 as $|z| \rightarrow 1$ since $f$ is in $\mathrm{VMO}_{\partial}(\mathbf{D})$. 
LEMMA 6. $\operatorname{ESV}(\mathbf{D}) \subset \tilde{Q}=\operatorname{VMO}_{\partial}(\mathbf{D}) \cap L^{\infty}(\mathbf{D})$.

Proof. Let $f \in \operatorname{ESV}(\mathbf{D})$. Then $|f|^{2} \in \operatorname{ESV}(\mathbf{D})$ since $\operatorname{ESV}(\mathbf{D})$ is a $C^{*}$ subalgebra of $L^{\infty}(\mathbf{D})$. By the corollary to Lemma 5 , we have $f(z)-\hat{f}(z) \rightarrow 0$ as $|z| \rightarrow 1^{-}, \widehat{|f|^{2}}(z)-|f|^{2}(z) \rightarrow 0$ as $|z| \rightarrow 1^{-}$. Moreover,

$$
\begin{aligned}
\left.|| \hat{f}(z)\right|^{2}-|f(z)|^{2} \mid & =(|\hat{f}(z)|+|f(z)|)|| \hat{f}(z)|-| f(z)|| \\
& \leq 2\|f\|_{\infty}|\hat{f}(z)-f(z)| \rightarrow 0 \quad \text { as }|z| \rightarrow 1^{-}
\end{aligned}
$$

So

$$
\widehat{|f|^{2}}(z)-|\hat{f}(z)|^{2}=\left(\widehat{|f|^{2}}(z)-|f(z)|^{2}\right)+\left(|f(z)|^{2}-|\hat{f}(z)|^{2}\right) \rightarrow 0 \quad \text { as }|z| \rightarrow 1^{-}
$$

and we have $f \in \mathrm{VMO}_{\partial}(\mathbf{D})$. Since $\operatorname{ESV}(\mathbf{D}) \subset L^{\infty}(\mathbf{D}), f \in \mathrm{VMO}_{\partial}(\mathbf{D}) \cap L^{\infty}(\mathbf{D})=$ $\tilde{Q}$.

Summarizing the above results, we have proved the following theorem.

THEOREM 5. $\tilde{Q}=\operatorname{ESV}(\mathbf{D})+\tilde{Q} \cap \tilde{B}$. A decomposition is given by $f=\hat{f}+(f-\hat{f})$.

COROLlaRY 1 .

$$
\operatorname{ESV}(\mathbf{D}) \cap \tilde{B}=\left\{f \in L^{\infty}(\mathbf{D}, d A) \mid f(z) \rightarrow 0 \text { as }|z| \rightarrow 1^{-}\right\} .
$$

ProOF. If $f(z) \rightarrow 0$ as $|z| \rightarrow 1^{-}$, then obviously $f \in \operatorname{ESV}(\mathbf{D})$ (just by definition). On the other hand,

$$
\tilde{f}(z)=\int_{\mathbf{D}} f\left(\frac{z-w}{1-\bar{z} w}\right) d A(w) \rightarrow 0 \quad\left(|z| \rightarrow 1^{-}\right)
$$

by the dominated convergence theorem. So $f \in \tilde{B}$, and hence $f \in \operatorname{ESV}(\mathbf{D}) \cap \tilde{B}$.

Conversely, if $f \in \operatorname{ESV}(\mathbf{D}) \cap \tilde{B}$, then $f(z)-\hat{f}(z) \rightarrow 0$ as $|z| \rightarrow 1^{-}$and

$$
\begin{aligned}
|\hat{f}(z)| & \leq \frac{1}{\left|S_{z}^{\prime}\right|} \int_{S_{z}^{\prime}}|f(w)| d A(w) \\
& \leq \frac{2}{\left|S_{z}\right|} \int_{S_{z}}|f(w)| d A(w) \rightarrow 0 \quad \text { as }|z| \rightarrow 1^{-}
\end{aligned}
$$

since $f \in \tilde{Q} \cap \tilde{B}$. Therefore, $f(z)=(f(z)-\hat{f}(z))+\hat{f}(z) \rightarrow 0$ as $|z| \rightarrow 1^{-}$.

COROllary 2. For $f \in \tilde{Q}=\operatorname{VMO}_{\partial}(\mathbf{D}) \cap L^{\infty}(\mathbf{D}), f \in \operatorname{ESV}(\mathbf{D})$ iff $f(z)-$ $\hat{f}(z) \rightarrow 0\left(|z| \rightarrow 1^{-}\right)$.

ProOF. The "only if" part follows from the corollary to Lemma 5. If $f(z)-$ $\hat{f}(z) \rightarrow 0$ as $|z| \rightarrow 1^{-}$, then $f-\hat{f} \in \operatorname{ESV}(\mathbf{D})$. So $f=(f-\hat{f})+\hat{f} \in \operatorname{ESV}(\mathbf{D})$.

THEOREM 6. If $f \in \operatorname{ESV}(\mathbf{D})$, then $f(z)-\tilde{f}(z) \rightarrow 0$ as $|z| \rightarrow 1^{-}$.

PROOF.

$$
\begin{gathered}
f(z)-\tilde{f}(z)=\left(1-|z|^{2}\right)^{2} \int_{\mathbf{D}} \frac{f(z)-f(w)}{|1-\bar{z} w|^{4}} d A(w), \\
|f(z)-\tilde{f}(z)| \leq\left(1-|z|^{2}\right)^{2} \int_{\mathbf{D}} \frac{|f(z)-f(w)|}{|1-\bar{z} w|^{4}} d A(w) .
\end{gathered}
$$


Given $\varepsilon>0$, by Lemma 3, there are $\sigma$ and $\delta_{0}$ in $(0,1)$ such that

$$
\int_{\mathrm{D}-S_{\delta e^{i \theta}}} \frac{\left(1-|z|^{2}\right)^{2}}{|1-\bar{z} w|^{4}} d A(w)<\varepsilon \quad\left(z=|z| e^{i \theta}\right)
$$

whenever $0<1-|z|<\delta_{0}$ and $1-|z|=(1-\delta) \sigma$. But $f \in \operatorname{ESV}(\mathbf{D})$, so there exists $\delta_{1} \in(0,1)$ such that

$$
\left|f\left(r_{1} e^{i \theta_{1}}\right)-f\left(r_{2} e^{i \theta_{2}}\right)\right|<\varepsilon \sigma^{2}
$$

whenever $r_{1}, r_{2} \in[1-\lambda, 1-\sigma \lambda], \lambda<\delta_{1}$, and $\left|\theta_{1}-\theta_{2}\right| \leq \max \left(1-r_{1}, 1-r_{2}\right)$.

Let $\delta_{2}=\min \left(\delta_{0}, \delta_{1}\right)$. Then for $0<1-|z|<\delta_{2}$ and $1-|z|=\sigma(1-\delta)$, we have

$$
\begin{aligned}
|f(z)-\tilde{f}(z)| \leq & 2\|f\|_{\infty} \int_{\mathbf{D}-S_{\delta e^{i \theta}}} \frac{\left(1-|z|^{2}\right)^{2}}{|1-\bar{z} w|^{4}} d A(w) \\
& +\left(1-|z|^{2}\right)^{2} \int_{S_{\delta e^{i \theta}}} \frac{|f(z)-f(w)|}{|1-\bar{z} w|^{4}} d A(w) \\
\leq & 2\|f\|_{\infty} \varepsilon+\frac{\left(1-|z|^{2}\right)^{2}}{(1-|z|)^{4}} \int_{S_{\delta e^{i \theta}}}|f(z)-f(w)| d A(w) \\
\leq & 2\|f\|_{\infty} \varepsilon+\frac{4}{(1-|z|)^{2}} \int_{S_{\delta e^{i \theta}}}\left|f(w)-f\left(\delta e^{i \theta}\right)\right| d A(w) \\
& +\frac{4}{(1-|z|)^{2}} \int_{S_{\delta e^{i \theta}}}\left|f(z)-f\left(\delta e^{i \theta}\right)\right| d A(w) .
\end{aligned}
$$

Since $\left|f(z)-f\left(\delta e^{i \theta}\right)\right|<\varepsilon \sigma^{2}$ by (4), and

$$
\frac{\left|S_{\delta e^{i \theta} \mid}\right|}{(1-|z|)^{2}}=\frac{(1+\delta)(1-\delta)^{2}}{(1-|z|)^{2}}=\frac{1+\delta}{\sigma^{2}} \leq \frac{2}{\sigma^{2}}
$$

we must have

$$
\begin{aligned}
|f(z)-\tilde{f}(z)| \leq 2\|f\|_{\infty} \varepsilon+\frac{\delta}{\sigma^{2}} \cdot \frac{1}{\left|S_{\delta e^{i \theta}}\right|} \int_{S_{\delta e^{i \theta}}}\left|f(w)-f\left(\delta e^{i \theta}\right)\right| d(w)+\delta \varepsilon \\
\left(0<1-|z|<\delta_{2}\right) .
\end{aligned}
$$

Because

$$
\lim _{|z| \rightarrow 1^{-}} \frac{1}{\left|S_{\delta e^{i \theta}}\right|} \int_{S_{\delta e^{i \theta}}}\left|f(w)-f\left(\delta e^{i \theta}\right)\right| d A(w)=0
$$

by Lemma 5 , we have

$$
\varlimsup_{|z| \rightarrow 1^{-}}|f(z)-\tilde{f}(z)| \leq 2\|f\|_{\infty} \varepsilon+\delta \varepsilon .
$$

This completes the proof of $\lim _{|z| \rightarrow 1^{-}}(f(z)-\tilde{f}(z))=0$ for any $f \in \operatorname{ESV}(\mathbf{D})$.

THEOREM 7. For $f \in \tilde{Q}=\mathrm{VMO}_{\partial}(\mathbf{D}) \cap L^{\infty}(\mathbf{D}, d A)$, we have

(1) $\tilde{f} \in \operatorname{ESV}(\mathbf{D})$;

(2) $f-\tilde{f} \in \tilde{Q} \cap \tilde{B}$.

Proof. (1). By Theorem 5, $f=f_{1}+f_{2}$, where $f_{1} \in \operatorname{ESV}(\mathbf{D})$ and $f_{2} \in \tilde{Q} \cap \tilde{B}$. Taking the Berezin symbol of $f$, we get $\tilde{f}=\tilde{f}_{1}+\tilde{f}_{2}$. 
Now $f_{2} \in \tilde{Q} \cap \tilde{B}$ implies $\tilde{f}_{2}(z) \rightarrow 0$ as $|z| \rightarrow 1^{-}$, so $\tilde{f}_{2} \in \operatorname{ESV}(\mathbf{D}) . f_{1} \in \operatorname{ESV}(\mathbf{D})$ implies $f_{1}(z)-\tilde{f}_{1}(z) \rightarrow 0$ as $|z| \rightarrow 1^{-}$, so $f_{1}-\tilde{f}_{1} \in \mathrm{ESV}$. Thus

$$
\tilde{f}_{1}=\left(\tilde{f}_{1}-f_{1}\right)+f_{1} \in \operatorname{ESV}(\mathbf{D}) .
$$

Hence

$$
\tilde{f}=\tilde{f}_{1}+\tilde{f}_{2} \in \operatorname{ESV}(\mathbf{D}) .
$$

(2). $f-\tilde{f}=f_{1}+f_{2}-\tilde{f}_{1}-\tilde{f}_{2}=\left(f_{1}-\tilde{f}_{1}\right)+f_{2}-\tilde{f}_{2} . f_{1}(z)-\tilde{f}_{1}(z) \rightarrow 0$ (as $\left.|z| \rightarrow 1^{-}\right)$implies $f_{1}-\tilde{f}_{1} \in \tilde{Q} \cap \tilde{B}$. $\tilde{f}_{2}(z) \rightarrow 0\left(\right.$ as $\left.|z| \rightarrow 1^{-}\right)$implies $\tilde{f}_{2} \in \tilde{Q} \cap \tilde{B}$. So $f-\tilde{f} \in \tilde{Q} \cap \tilde{B}$.

COROLlaRY 1. If $f \in \tilde{Q}$, then $\tilde{f}(z)-\hat{f}(z) \rightarrow 0$ as $|z| \rightarrow 1^{-}$.

Proof. If $f \in \tilde{Q}$, then $\tilde{f}$ and $\hat{f}$ are $\operatorname{ESV}(\mathbf{D})$ by Theorems 3 and 7 , so $\tilde{f}-\hat{f}$ are $\operatorname{ESV}(\mathbf{D})$. On the other hand,

$$
\tilde{f}-\hat{f}=(\tilde{f}-f)+(f-\hat{f}) \in \tilde{Q} \cap \tilde{B}
$$

by Theorems 4 and 7 . Therefore,

$$
\tilde{f}-\hat{f} \in \operatorname{ESV}(\mathbf{D}) \cap \tilde{Q} \cap \tilde{B}=\operatorname{ESV}(\mathbf{D}) \cap \tilde{B} .
$$

Applying Corollary 1 to Theorem 5, we get $\tilde{f}(z)-\hat{f}(z) \rightarrow 0$ as $|z| \rightarrow 1^{-}$.

COROLlary 2. For $f \in \tilde{Q}$, we have $f \in \operatorname{ESV}(\mathbf{D})$ iff $f(z)-\tilde{f}(z) \rightarrow 0$ as $|z| \rightarrow 1$.

Proof. If $f \in \operatorname{ESV}(\mathbf{D})$, then $f(z)-\tilde{f}(z) \rightarrow 0(|z| \rightarrow 1)$ by Theorem 6. If $f(z)-\tilde{f}(z) \rightarrow 0$, then $f-\tilde{f} \in \operatorname{ESV}(\mathbf{D})$, but $\tilde{f} \in \operatorname{ESV}(\mathbf{D})$ by Theorem 7 , so $f=(f-\tilde{f})+\tilde{f} \in \operatorname{ESV}(\mathbf{D})$.

REMARK. For the identity $\tilde{Q}=\operatorname{ESV}(\mathbf{D})+\tilde{Q} \cap \tilde{B}$, we have found two canonical decompositions:

$$
f=\tilde{f}+(f-\tilde{f}) \quad \text { and } \quad f=\hat{f}+(f-\hat{f}) .
$$

THEOREM 8. For $f \in L^{\infty}(\mathbf{D})$, we have $f \in \operatorname{ESV}(\mathbf{D})$ iff $\left\|f(z)-f \circ b_{z}\right\|_{L^{2}} \rightarrow 0$ as $|z| \rightarrow 1^{-}$, where $b_{z}(w)=(z-w) /(1-\bar{z} w)$, and the norm is just the usual $L^{2}$-norm.

ProOF. For $f \in L^{\infty}(\mathbf{D})$, it is easy to check the following identity:

$$
\left\|f(z)-f \circ b_{z}\right\|_{L^{2}}^{2}=\widetilde{|f|^{2}}(z)-|\tilde{f}(z)|^{2}+|\tilde{f}(z)-f(z)|^{2} .
$$

If the left-hand side of (5) goes to 0 as $|z| \rightarrow 1^{-}$, then $\widetilde{|f|^{2}}(z)-|\tilde{f}(z)|^{2} \rightarrow 0(|z| \rightarrow$ 1) and $|\tilde{f}(z)-f(z)| \rightarrow 0$. The first limit says that $f$ is in $\tilde{Q}$, the second limit and Corollary 2 to Theorem 7 imply that $f$ in in $\operatorname{ESV}(D)$.

Conversely, if $f \in \operatorname{ESV}(\mathbf{D}) \subset \tilde{Q}$, the $\widetilde{|f|^{2}}(z)-|\tilde{f}(z)|^{2} \rightarrow 0$ and $|f(z)-\tilde{f}(z)| \rightarrow 0$ as $|z| \rightarrow 1^{-}$, so the left-hand side of (5) goes to 0 as $|z| \rightarrow 1^{-}$.

LEMMA 7. There is an absolute constant $C$ such that

$$
\int_{\mathbf{D}}|f(z)-f(0)|^{2} d A(z) \leq C \int_{\mathbf{D}}\left(1-|z|^{2}\right)^{2}\left|f^{\prime}(z)\right|^{2} d A(z)
$$

for all $f \in H^{\infty}(\mathbf{D})$. 
ProOF. Using Green's formula, we can easily prove (see p. 236 of [13])

$$
\int_{|z|<r}\left|f^{\prime}(z)\right|^{2} \log \frac{r}{|z|} d A(z)=\frac{1}{4 \pi} \int_{0}^{2 \pi}\left|f\left(r e^{i \theta}\right)-f(0)\right|^{2} d \theta
$$

It is also known (p. 237 of $[\mathbf{1 3}])$ that

$$
\int_{|z|<r}\left|f^{\prime}(z)\right|^{2} \log \frac{r}{|z|} d A(z) \leq C \int_{|z|<r}\left|f^{\prime}(z)\right|^{2}\left(1-\left|\frac{z}{r}\right|^{2}\right) d A(z)
$$

for all $f \in H^{\infty}(\mathbf{D})$, where $C$ is an absolute constant, i.e. $C$ does not depend on $f$. Now integrating the above inequality with respect to $r d r$, we get

$$
\frac{1}{4 \pi} \int_{0}^{1} r d r \int_{0}^{2 \pi}\left|f\left(r e^{i \theta}\right)-f(0)\right|^{2} d \theta \leq C \int_{0}^{1} r d r \int_{|z|<r}\left|f^{\prime}(z)\right|^{2}\left(1-\left|\frac{z}{r}\right|^{2}\right) d A(z),
$$

or

$$
\frac{1}{4} \int_{\mathbf{D}}|f(z)-f(0)|^{2} d A(z) \leq \frac{C}{2} \int_{\mathbf{D}}\left[1-|z|^{2}+|z|^{2} \log |z|^{2}\right]\left|f^{\prime}(z)\right|^{2} d A(z) .
$$

Power series expansion shows that

$$
1-|z|^{2}+|z|^{2} \log |z|^{2} \leq\left(1-|z|^{2}\right)^{2}
$$

so we have

$$
\int_{\mathbf{D}}|f(z)-f(0)|^{2} d A(z) \leq 2 C \int_{\mathbf{D}}\left|f^{\prime}(z)\right|^{2}\left(1-|z|^{2}\right)^{2} d A(z) .
$$

THEOREM 9. For $f \in H^{\infty}(\mathbf{D})$, the following are all equivalent:

(1) $f \in \operatorname{ESV}(\mathbf{D})$;

(2) $f \in \mathrm{VMO}_{\partial}(\mathbf{D})$;

(3) $f \in \tilde{Q}$;

(4) $f \in B_{0}$, where $B_{0}$ is the "little Bloch" space consisting of all the analytic functions $g$ on $\mathbf{D}$ such that $\left|g^{\prime}(z)\right|\left(1-|z|^{2}\right) \rightarrow 0$ as $|z| \rightarrow 1^{-}$.

ProOF. (2) and (3) are equivalent by Theorem 1. That (1) implies (3) follows from the fact that $\operatorname{ESV}(\mathbf{D}) \subset \tilde{Q}$. If $f \in \tilde{Q}$, then

$$
\left\|f(z)-f \circ b_{z}\right\|_{L^{2}}^{2}=\widetilde{|f|^{2}}(z)-|\tilde{f}(z)|^{2}+|\tilde{f}(z)-f(z)|^{2} \rightarrow 0
$$

as $|z| \rightarrow 1^{-}$since $\tilde{f}=f$ for $f \in H^{\infty}(\mathbf{D})$, so $f \in \operatorname{ESV}(\mathbf{D})$ by Theorem 8. Thus we have proved that (3) implies (1).

Next we prove the equivalence of (3) and (4).

If we replace $f$ by $f \circ b_{z_{0}}$ in Lemma 7 , then the inequality becomes

$$
\widetilde{|f|^{2}}\left(z_{0}\right)-\left|\tilde{f}\left(z_{0}\right)\right|^{2} \leq C \int_{\mathbf{D}}\left(1-\left|\frac{z_{0}-z}{1-\bar{z}_{0} z}\right|^{2}\right)^{2}\left|f^{\prime}\left(\frac{z_{0}-z}{1-\bar{z}_{0} z}\right)\right|^{2} d A(z) .
$$

Now if $f \in B_{0}$, then

$$
\left(1-\left|\frac{z_{0}-z}{1-\bar{z}_{0} z}\right|^{2}\right)^{2}\left|f^{\prime}\left(\frac{z_{0}-z}{1-\bar{z}_{0} z}\right)\right|^{2} \rightarrow 0
$$


as $\left|z_{0}\right| \rightarrow 1^{-}$for any fixed $z \in \mathbf{D}$. Thus by the dominated convergence theorem, we have $\widetilde{|f|^{2}}\left(z_{0}\right)-\left|\tilde{f}\left(z_{0}\right)\right|^{2} \rightarrow 0$ as $\left|z_{0}\right| \rightarrow 1^{-}$. This shows that (4) implies (3).

To prove (3) implies (4), we use the Bergman formula

$$
f(z)-f(0)=\int_{\mathbf{D}} \frac{f(w)-f(0)}{(1-z \bar{w})^{2}} d A(w), \quad f \in H^{\infty}(\mathbf{D}) .
$$

Taking derivative on both sides, we get

$$
f^{\prime}(z)=\int_{\mathbf{D}} \frac{2 \bar{w}(f(w)-f(0))}{(1-z \bar{w})^{3}} d A(w) .
$$

Let $z=0$, then

$$
\left|f^{\prime}(0)\right|^{2} \leq 4 \int_{\mathbf{D}}|f(w)-f(0)|^{2} d A(w), \quad f \in H^{\infty}(\mathbf{D})
$$

Replacing $f$ by $f \circ b_{z}$, we get

$$
\left|f^{\prime}(z)\right|^{2}\left(1-|z|^{2}\right)^{2} \leq 4\left(\widetilde{|f|^{2}}(z)-|\tilde{f}(z)|^{2}\right), \quad z \in \mathbf{D}
$$

This completes the proof of Theorem 9.

5. Symbol calculus of Toeplitz operators. In this section, we are going to determine the largest $C^{*}$-subalgebra $Q$ of $L^{\infty}(\mathbf{D}, d A)$ such that the map $\xi: Q \rightarrow$ $B\left(L_{a}^{2}(\mathbf{D})\right) / \mathcal{K}$, defined by $\xi(f)=T_{f}+\mathcal{K}$, is a $C^{*}$-algebra homomorphism, where $\mathcal{K}$ is the compact ideal of the full algebra $B\left(L_{a}^{2}(\mathbf{D})\right)$ of bounded linear operators on $L_{a}^{2}(\mathbf{D})$. First we establish the existence of such an algebra.

Let

$$
\begin{aligned}
& \Gamma=\left\{f \in L^{\infty}(\mathbf{D}) \mid T_{g} T_{f}-T_{g f} \in \mathcal{K} \text { for all } g \in L^{\infty}(\mathbf{D})\right\} \\
& Q=\Gamma \cap \bar{\Gamma} \\
& B=\left\{f \in L^{\infty}(\mathbf{D}) \mid T_{f} \in \mathcal{K}\right\}
\end{aligned}
$$

Proposition 5. For $f \in L^{\infty}(\mathbf{D})$, the following are all equivalent:

(1) $f \in \Gamma$;

(2) $H_{f}$ is compact;

(3) $T_{|f|^{2}}-T_{\bar{f}} T_{f}$ is compact.

ProOF. The proof is the same as in [6].

Proposition 6. For $f \in L^{\infty}(\mathbf{D})$, the following are all equivalent:

(1) $f \in Q$;

(2) $H_{f}$ and $H_{\bar{f}}$ are compact;

(3) $T_{|f|^{2}}-T_{f} T_{\bar{f}}$ and $T_{|f|^{2}}-T_{\bar{f}} T_{f}$ are compact.

PrOOF. The proof follows from Proposition 5.

Proposition 7. $Q$ is a $C^{*}$-subalgebra of $L^{\infty}(\mathbf{D}) ; Q \cap B$ is a closed selfadjoint ideal of $Q$.

PROOF. The proof is the same as in [6] and [7].

REMARK. Propositions 6 and 7 imply that $Q$ is the largest $C^{*}$-subalgebra of $L^{\infty}(\mathbf{D})$ such that the map $\xi: Q \rightarrow B\left(L_{a}^{2}(\mathbf{D})\right) / \mathcal{K}$ is a $C^{*}$-algebra homomorphism. 
The kernel of this homomorphism is $Q \cap B$. Thus if we let $\tau(Q)$ denote the $C^{*}$ subalgebra of $B\left(L_{a}^{2}(\mathbf{D})\right)$ generated by all the operators $T_{f}$ with $f \in Q$, then

$$
Q / Q \cap B \cong \tau(Q) / K
$$

as $C^{*}$-algebras. (6) is traditionally called the symbol calculus of Toeplitz operators. So far $Q$ has only been defined abstractly. Next we want to determine $Q$. The main theorem is that for a function $f \in L^{\infty}(\mathbf{D}) . f \in Q$ if and only if $f \in \mathrm{VMO}_{\partial}(\mathbf{D})$; $f \in Q \cap B$ if and only if $f \in \tilde{Q} \cap \tilde{B}$.

Proposition 8 (C. A. Berger). The operator $P: L^{\infty}(\mathbf{D}) \rightarrow L_{a}^{2}(\mathbf{D})$ is compact.

ProOF. Given a bounded sequence $\left\{f_{n}\right\}$ in $L^{\infty}(\mathbf{D})$, say, $\left\|f_{n}\right\|_{\infty} \leq M(n=$ $1,2, \ldots)$. We want to find a subsequence $\left\{f_{n_{k}}\right\}$ such that $\left\{P f_{n_{k}}\right\}$ converges in $L_{a}^{2}(\mathrm{D})$.

Recall that

$$
P f_{n}(z)=\int_{\mathbf{D}} \frac{f_{n}(w)}{(1-z \bar{w})^{2}} d A(w), \quad z \in \mathbf{D} .
$$

Now if $|z| \leq \delta<1$, then

$$
\left|P f_{n}(z)\right| \leq \int_{\mathbf{D}} \frac{M d A(w)}{|1-z \bar{w}|^{2}} \leq \frac{M}{(1-\delta)^{2}}, \quad n=1,2, \ldots
$$

So $\left\{P f_{n}\right\}$ is uniformly bounded on every compact subset of D. Since $\left\{P f_{n}\right\}$ is a sequence of analytic functions on $\mathbf{D}$, by Arzela's theorem, there is a subsequence $\left\{P f_{n_{k}}\right\}$ which converges to $h \in L_{a}^{2}(\mathbf{D})$ uniformly on every compact subset of $\mathbf{D}$. (Note: $h \in L_{a}^{2}(\mathbf{D})$ by Fatou's lemma.) It remains to prove that

$$
\left\|P f_{n_{k}}-h\right\|_{L^{2}} \rightarrow 0 \quad(k \rightarrow+\infty)
$$

For any $z \in \mathbf{D}$, we have

$$
\left|P f_{n}(z)\right| \leq M \int_{\mathbf{D}} \frac{d A(w)}{|1-z \bar{w}|^{2}}=-\frac{M}{2|z|^{2}} \ln \left(1-|z|^{2}\right) .
$$

Since $\int_{\mathbf{D}}\left(\frac{1}{2}|z|^{-2} \ln \left(1-|z|^{2}\right)\right)^{2} d A(z)<+\infty,(7)$ follows from the dominated convergence theorem.

LEMMA 8. If $\left\{f_{n}\right\}$ is a sequence of real-valued functions in $L^{2}(\mathbf{D})$ such that $\left\|f_{n}-h\right\|_{L^{2}} \rightarrow 0(n \rightarrow+\infty)$ for some $h \in L_{a}^{2}(\mathbf{D})$, then $h$ is a constant.

Proof. Write $h=u+i v$. Then

$$
\left|f_{n}(z)-h(z)\right|^{2}=\left(f_{n}(z)-u(z)\right)^{2}+(v(z))^{2},
$$

so

$$
\left\|f_{n}-h\right\|_{L^{2}}^{2}=\left\|f_{n}-u\right\|_{L^{2}}^{2}+\|v\|_{L^{2}}^{2} \geq\|v\|_{L^{2}}^{2} .
$$

Let $n \rightarrow+\infty$, we have $v=0$. Thus $h$ is real-valued. Since $h$ is analytic, $h$ must be a constant. 
THEOREM 10. $Q \subset \tilde{Q}=\mathrm{VMO}_{\partial}(\mathbf{D}) \cap L^{\infty}(\mathbf{D})$.

Proof. Given $f \in Q$, we want to prove $\widetilde{|f|^{2}}(z)-|\tilde{f}(z)|^{2} \rightarrow 0\left(|z| \rightarrow 1^{-}\right)$. Since $Q$ and $\tilde{Q}$ are selfadjoint, we might as well assume that $f$ is real-valued.

It is easy to check that

$$
\widetilde{|f|^{2}}(z)-|\tilde{f}(z)|^{2}=\left\|\tilde{f}(z)-f \circ b_{z}\right\|_{L^{2}}^{2} \geq 0,
$$

where $b_{z}(w)=(z-w) /(1-\bar{z} w)$. We prove the theorem by contradiction.

Suppose

$$
\varlimsup_{|z| \rightarrow 1^{-}}\left\|\tilde{f}(z)-f \circ b_{z}\right\|^{2}>0 .
$$

Then there exists $\rho>0$ and $\left|z_{n}\right| \rightarrow 1^{-}$such that

$$
\left\|\tilde{f}\left(z_{n}\right)-f \circ b_{z_{n}}\right\|^{2}>\rho, \quad n=1,2, \ldots
$$

Because $f \in Q, H_{f}=(I-P) M_{f} P$ is compact, so

$$
\left\|(I-P) f k_{z}\right\|_{L^{2}} \rightarrow 0 \quad\left(|z| \rightarrow 1^{-}\right)
$$

since $k_{z} \rightarrow 0$ weakly as $|z| \rightarrow 1^{-}$.

For each $z \in \mathbf{D}$, define a unitary operator $U_{z}$ on $L^{2}(\mathbf{D})$ as follows:

$$
U_{z} f(w)=\frac{1-|z|^{2}}{(1-\bar{z} w)^{2}} f\left(\frac{z-w}{1-\bar{z} w}\right), \quad w \in \mathbf{D}, f \in L^{2}(\mathbf{D}) .
$$

It is easy to check that $U_{z}^{*}=U_{z}$ and $L_{a}^{2}(\mathbf{D})$ is a reducing subspace of $U_{z}$, so $U_{z} P=P U_{z}$.

Now using the equality $f k_{z}=U_{z}\left(f \circ b_{z}\right)$ and (9), we get

$$
\left\|(I-P) U_{z}\left(f \circ b_{z}\right)\right\|_{L^{2}} \rightarrow 0 \quad\left(\text { as }|z| \rightarrow 1^{-}\right) .
$$

But $(I-P) U_{z}=U_{z}(I-P)$ and $U_{z}$ is unitary, so we must have

$$
\left\|(I-P) f \circ b_{z}\right\|_{L^{2}} \rightarrow 0 \quad\left(\text { as }|z| \rightarrow 1^{-}\right) .
$$

Notice that $\left\|f \circ b_{z}\right\|_{\infty}=\|f\|_{\infty}$ for all $z \in \mathbf{D}$, so by Proposition 8, there is a subsequence $\left\{z_{n_{k}}\right\}$ of $\left\{z_{n}\right\}$ and $h \in L_{a}^{2}(\mathbf{D})$ such that

$$
\left\|P\left(f \circ b_{z_{n_{k}}}\right)-h\right\|_{L^{2}} \rightarrow 0 \quad(k \rightarrow+\infty) .
$$

Now $(10)+(11)$ implies that

$$
\left\|f \circ b_{z_{n_{k}}}-h\right\|_{L^{2}} \rightarrow 0 \quad(k \rightarrow+\infty) .
$$

By Lemma $8, h$ is a constant. Therefore,

$$
\tilde{f}\left(z_{n_{k}}\right)=\left\langle f \circ b_{z_{n_{k}}}, 1\right\rangle \rightarrow\langle h, 1\rangle=h
$$

as $k \rightarrow+\infty$. Thus

$$
\begin{aligned}
\left\|f \circ b_{z_{n_{k}}}-\tilde{f}\left(z_{n_{k}}\right)\right\|_{L^{2}} & \leq\left\|f \circ b_{z_{n_{k}}}-h\right\|_{L^{2}}+\left\|h-\tilde{f}\left(z_{n_{k}}\right)\right\|_{L^{2}} \\
& =\left\|f \circ b_{z_{n_{k}}}-h\right\|_{L^{2}}+\left|\tilde{f}\left(z_{n_{k}}\right)-h\right| \rightarrow 0 \quad \text { as } k \rightarrow+\infty
\end{aligned}
$$

a contradiction to (8).

REMARK. The proof of Theorem 10 is a modification of the corresponding result in an early version of [7]. 
Proposition 9. Let

$$
M_{p}=\sup _{z \in \mathbf{D}} \int_{\mathbf{D}} \frac{d A(w)}{|1-\bar{z} w|^{p}\left(1-|w|^{2}\right)^{p / 2}} .
$$

Then $M_{p}<+\infty$ for $p<\frac{4}{3}$.

PrOOF. See [1], or 1.4 .10 of [21].

LeMmA 9. Let $\mathbf{D}_{\delta}=\{z \in \mathbf{D}|| z \mid<\delta\}, \delta \in(0,1)$. Then $M_{\chi_{\mathbf{D}_{\delta}}} H_{f}$ is HilbertSchmidt as an operator from $L_{a}^{2}(\mathbf{D})$ to $L^{2}(\mathbf{D})$ for all $f$ in $L^{\infty}(\mathbf{D})$.

PROOF. For $|z| \geq \delta, M_{\chi_{\mathbf{D}_{\delta}}} H_{f} g(z)=0$. For $|z|<\delta$,

$$
M_{\chi_{\mathbf{D}_{\delta}}} H_{f} g(z)=H_{f} g(z)=\int_{\mathbf{D}} \frac{f(z)-f(w)}{(1-z \bar{w})^{2}} g(w) d A(w) .
$$

Thus for all $z \in \mathbf{D}$ and $g \in L_{a}^{2}(\mathbf{D})$,

$$
\left|M_{\chi_{\mathbf{D}_{\delta}}} H_{f} g(z)\right| \leq \int_{\mathbf{D}} \frac{|f(z)-f(w)|}{(1-\delta)^{2}}|g(w)| d A(w) .
$$

The operator $A$ on $L^{2}(\mathbf{D})$ defined by

$$
A g(z)=\int_{\mathbf{D}}|f(z)-f(w)| g(w) d A(w)
$$

is Hilbert-Schmidt since the integral kernel is in $L^{2}(\mathbf{D} \times \mathbf{D})$, so $M_{\chi_{\mathbf{D}_{\delta}}} H_{f}$ is HilbertSchmidt by (13).

THEOREM 11. $\operatorname{ESV}(\mathbf{D}) \subset Q$.

Proof. Let $f \in \operatorname{ESV}(\mathbf{D})$. Then by Theorem $8,\left\|f(z)-f \circ b_{z}\right\|_{L^{2}} \rightarrow 0$ as $|z| \rightarrow 1^{-}$. Given $\varepsilon>0$, choose $\delta \in(0,1)$ such that $\left\|f(z)-f \circ b_{z}\right\|_{L^{2}}<\varepsilon^{3}$ for all $\delta \leq|z|<1$. Then for all $\delta \leq|z|<1$, we have

$$
\begin{gathered}
\int_{\mathbf{D}} \frac{|f(z)-f(w)|}{|1-z \bar{w}|^{2} \sqrt{1-|w|^{2}}} d A(w)=\frac{1}{\sqrt{1-|z|^{2}}} \int_{\mathbf{D}} \frac{\left|f(z)-f \circ b_{z}(w)\right|}{|1-\bar{z} w| \sqrt{1-|w|^{2}}} d A(w) \\
\leq \frac{M}{\sqrt{1-|z|^{2}}}\left(\int_{\mathbf{D}}\left|f(z)-f \circ b_{z}(w)\right|^{6} d A(w)\right)^{1 / 6} \\
\leq \frac{M\left(2\|f\|_{\infty}\right)^{2 / 3}}{\sqrt{1-|z|^{2}}}\left(\int_{\mathbf{D}}\left|f(z)-f \circ b_{z}(w)\right|^{2} d A(w)\right)^{1 / 6} \\
=\frac{M\left(2\|f\|_{\infty}\right)^{2 / 3}}{\sqrt{1-|z|^{2}}}\left\|f(z)-f \circ b_{z}\right\|_{L^{2}}^{1 / 3}<\frac{M\left(2\|f\|_{\infty}\right)^{2 / 3} \varepsilon}{\sqrt{1-|z|^{2}}}
\end{gathered}
$$

where $M=M_{6 / 5}$ in Proposition 9 .

It is easy to check that

$$
H_{f} g(z)=\int_{\mathbf{D}} \frac{f(z)-f(w)}{(1-z \bar{w})^{2}} g(w) d A(w), \quad g \in L_{a}^{2} .
$$

So

$$
\left|H_{f} g(z)\right| \leq \int_{\mathbf{D}} \frac{|f(z)-f(w)|}{|1-z \bar{w}|^{2}}|g(w)| d A(w)
$$


The Cauchy-Schwarz inequality shows that

$$
\begin{aligned}
\left|H_{f} g(z)\right|^{2} \leq & \int_{\mathbf{D}} \frac{|f(z)-f(w)|}{|1-z \bar{w}|^{2} \sqrt{1-|w|^{2}}} d A(w) \\
& \cdot \int_{\mathbf{D}} \frac{|f(z)-f(w)|}{|1-z \bar{w}|^{2}} \sqrt{1-|w|^{2}}|g(w)|^{2} d A(w) .
\end{aligned}
$$

Thus for all $\delta \leq|z|<1$,

$$
\begin{aligned}
\left|H_{f} g(z)\right|^{2} & \leq \frac{M\left(2\|f\|_{\infty}\right)^{2 / 3}}{\sqrt{1-|z|^{2}}} \varepsilon \int_{\mathbf{D}} \frac{|f(z)-f(w)|}{|1-z \bar{w}|^{2}} \sqrt{1-|w|^{2}}|g(w)|^{2} d A \\
& \leq \frac{M\left(2\|f\|_{\infty}\right)^{5 / 3}}{\sqrt{1-|z|^{2}}} \varepsilon \int_{\mathbf{D}} \frac{\sqrt{1-|w|^{2}}|g(w)|^{2}}{|1-z \bar{w}|^{2}} d A(w) .
\end{aligned}
$$

Write $\bar{M}=M\left(2\|f\|_{\infty}\right)^{5 / 3}$. Then

$$
\begin{aligned}
\int_{|z| \geq \delta} & \left|H_{f} g(z)\right|^{2} d A(z) \\
& \leq \bar{M} \varepsilon \int_{\mathbf{D}} \sqrt{1-|w|^{2}}|g(w)|^{2} d A(w) \cdot \int_{\mathbf{D}} \frac{d A(z)}{\sqrt{1-|z|^{2}}|1-\bar{w} z|^{2}} \\
& =\bar{M} \varepsilon \int_{\mathbf{D}}|g(w)|^{2} d A(w) \cdot \int_{\mathbf{D}} \frac{d A(z)}{|1-\bar{w} z| \sqrt{1-|z|^{2}}} \\
& \leq \bar{M} M_{1} \varepsilon \int_{\mathbf{D}}|g(w)|^{2} d A(w)=\bar{M} M_{1} \varepsilon\|g\|^{2} .
\end{aligned}
$$

This implies that $\left\|M_{\chi_{\mathbf{D}-\mathbf{D}_{\delta}}} H_{f}\right\| \leq \bar{M} M_{1} \varepsilon$, namely, $\left\|H_{f}-M_{\chi_{\mathbf{D}_{\delta}}} H_{f}\right\| \leq \bar{M} M_{1} \varepsilon$. Since $M_{\chi_{\mathbf{D}_{\delta}}} H_{f}$ is compact and $\varepsilon$ is arbitrary, $H_{f}$ is compact, and so $f \in \Gamma$. Because $f$ is arbitrary and $\operatorname{ESV}(\mathbf{D})$ is selfadjoint, we have proved $\operatorname{ESV}(\mathbf{D}) \subset \Gamma \cap \bar{\Gamma}=Q$.

THEOREM 12. $\tilde{Q} \cap \tilde{B} \subset Q \cap B$.

Proof. Given $f \in \tilde{Q} \cap \tilde{B}$, we have $|f| \in \tilde{B}$. To prove $f \in Q \cap B$, it suffices to prove $|f| \in B$, that is, $T_{|f|}$ is compact.

Recall that

$$
T_{|f|} g(z)=\int_{\mathbf{D}} \frac{|f(w)|}{(1-z \bar{w})^{2}} g(w) d A(w)
$$

for $g \in L_{a}^{2}(\mathbf{D})$ and $z \in \mathbf{D}$. So the Cauchy-Schwarz inequality gives

$$
\left|T_{|f|} g(z)\right|^{2} \leq \int_{\mathbf{D}} \frac{|f(w)|^{2} d A(w)}{|1-z \bar{w}|^{2} \sqrt{1-|w|^{2}}} \int_{\mathbf{D}} \frac{\sqrt{1-|w|^{2}}|g(w)|^{2}}{|1-z \bar{w}|^{2}} d A(w)
$$

But

$$
\begin{aligned}
\int_{\mathbf{D}} \frac{|f(w)|^{2} d A(w)}{|1-z \bar{w}|^{2} \sqrt{1-|w|^{2}}}= & \frac{1}{\sqrt{1-|z|^{2}}} \int_{\mathbf{D}} \frac{\left|f \circ b_{z}(w)\right|^{2}}{|1-z \bar{w}| \sqrt{1-|w|^{2}}} d A(w) \\
& \leq \frac{M_{6 / 5}}{\sqrt{1-|z|^{2}}}\left(\int_{\mathbf{D}}\left|f \circ b_{z}(w)\right|^{12} d A(w)\right)^{1 / 6} \\
& \leq \frac{M_{6 / 5}\|f\|_{\infty}^{11 / 6}}{\sqrt{1-|z|^{2}}}\left(\int_{\mathbf{D}}\left|f \circ b_{z}(w)\right| d A(w)\right)^{1 / 6}
\end{aligned}
$$


and we have

$$
\left|T_{|f|} g(z)\right|^{2} \leq \frac{\bar{M}}{\sqrt{1-|z|^{2}}}(\widetilde{|f|} \mid(z))^{1 / 6} \int_{\mathbf{D}} \frac{\sqrt{1-|w|^{2}}|g(w)|^{2}}{|1-z \bar{w}|^{2}} d A(w)
$$

where $\bar{M}=M_{6 / 5}\|f\|_{\infty}^{11 / 6}$.

Given $\varepsilon>0$, choose $\delta \in(0,1)$ such that $\widetilde{|f|} \mid(z)<\varepsilon^{6}$ whenever $\delta<|z|<1$. Then

$$
\begin{aligned}
\int_{|z| \geq \delta}\left|T_{|f|} g(z)\right|^{2} d A(z) \leq & \bar{M} \varepsilon \int_{\mathbf{D}}|g(w)|^{2} \sqrt{1-|w|^{2}} d A(w) \\
& \cdot \int_{\mathbf{D}} \frac{d A(z)}{|1-z \bar{w}|^{2} \sqrt{1-|z|^{2}}} \\
= & \bar{M} \varepsilon \int_{\mathbf{D}}|g(w)|^{2} d A(w) \int_{\mathbf{D}} \frac{d A(z)}{|1-z \bar{w}| \sqrt{1-|z|^{2}}} \\
\leq & \bar{M} M_{1} \varepsilon \int_{\mathbf{D}}|g(w)|^{2} d A(w)=\bar{M} M_{1} \varepsilon\|g\|_{L^{2}}^{2} .
\end{aligned}
$$

So $\left\|M_{\chi \mathbf{D}-\mathbf{D}_{\delta}} T_{|f|}\right\|^{2} \leq \bar{M} M_{1} \varepsilon$, that is,

$$
\left\|T_{|f|}-M_{\chi \mathbf{D}_{\delta}} T_{|f|}\right\|^{2} \leq \bar{M} M_{1} \varepsilon .
$$

Since $M_{\chi_{\mathbf{D}_{\delta}}} T_{|f|}$ is compact as an operator from $L_{a}^{2}(\mathbf{D})$ to $L^{2}(\mathbf{D})$ and $\varepsilon$ is arbitrary, $T_{|f|}$ must be compact.

REMARK. Since $k_{z} \rightarrow 0$ weakly as $|z| \rightarrow 1^{-}$, we have $Q \cap B \subset \tilde{Q} \cap \tilde{B}$ trivially.

Theorems 11 and 12 and the decomposition $\tilde{Q}=\operatorname{ESV}(\mathbf{D})+\tilde{Q} \cap \tilde{B}$ show that $\tilde{Q} \subset Q$. In summary, we have proved the following main theorem.

THEOREM 13. (1) $Q=\tilde{Q}=\operatorname{VMO}_{\partial}(\mathbf{D}) \cap L^{\infty}(\mathbf{D})$.

(2) $Q \cap B=\tilde{Q} \cap \tilde{B}$.

COROllary 1 (S. AXler [1]). Let $f \in H^{\infty}(\mathbf{D})$. Then $H_{\bar{f}}$ is compact if and only if $f$ is in the "little Bloch" space $B_{0}$.

ProOF. It follows from Theorems 9 and 13 and the fact that $H_{f}=0$.

COROLlaRY 2. $Q$ and $Q \cap B$ are invariant under Möbius transformations.

ProOF. This follows from the facts that $Q=\tilde{Q}$ and $Q \cap B=\tilde{Q} \cap \tilde{B}$ and $\tilde{f}\left(b_{\lambda}(z)\right)=\widetilde{f \circ b_{\lambda}}(z)$ (simply a change of variable formula), where the $b_{\lambda}$ 's are Möbius transformations.

6. Fredholm theory of Toeplitz operators with symbols in $Q$. The isomorphism $Q / Q \cap B \cong \tau(Q) / \mathcal{K}$ and the decomposition $Q=\mathrm{ESV}+Q \cap B$ will serve as basic tools for our study of Fredholm theory of Toeplitz operators with symbols in $Q$. Let $\mathrm{BC}(\mathbf{D})$ be the $C^{*}$-algebra of all bounded continuous functions on $\mathbf{D}$, and $C_{0}(\mathbf{D})$ be the space of continuous functions $f$ on $\mathbf{D}$ with the property that $f(z) \rightarrow 0$ as $|z| \rightarrow 1^{-}$. Consider the algebra BCESV defined as BC(D) $\cap$ ESV. Since $\tilde{f} \in \mathrm{BC}(\mathbf{D})$ for any $f \in L^{\infty}(\mathbf{D})$, the equality $f=\tilde{f}+(f-\tilde{f})$ gives a decomposition

$$
Q=\mathrm{BCESV}+Q \cap B
$$


Notice that $\mathrm{BCESV} \cap(Q \cap B)=C_{0}(\mathbf{D})$, so we have

$$
Q / Q \cap B=(\mathrm{BCESV}+Q \cap B) / Q \cap B \cong \mathrm{BCESV} / C_{0}(\mathbf{D})
$$

Also we should mention that

$$
Q / Q \cap B \cong \mathrm{ESV} / V_{0}(\mathbf{D})
$$

where $V_{0}(\mathbf{D})$ consists of all functions $f$ in $L^{\infty}(\mathbf{D})$ with $f(z) \rightarrow 0$ as $|z| \rightarrow 1^{-}$.

Let $[23 \beta \beta \mathrm{D}$ be the Stone-Cerch compactification of $\mathbf{D}$. Any bounded continuous functions $f$ on $\mathbf{D}$ has a unique continuous extension to $\beta \mathbf{D}$ : we also denote this extension of $f$ to $\beta \mathrm{D}$ by $f$, so there should be no confusion about this.

THEOREM 14. If $f \in Q$, then $\sigma_{e}\left(T_{f}\right)=\tilde{f}(\beta \mathbf{D}-\mathbf{D})$, where $\sigma_{e}\left(T_{f}\right)$ is the essential spectrum of $T_{f}$.

Proof. Since $f \in Q$, we know $T_{f-\tilde{f}}$ is compact. Thus $\sigma_{e}\left(T_{f}\right)=\sigma_{e}\left(T_{\tilde{f}}\right) . \tilde{f}$ is in BCESV. Mimicking [7], we can prove that for any $g \in \mathrm{BCESV}, g+C_{0}(\mathbf{D})$ is invertible in BCESV $/ C_{0}(\mathbf{D})$ if and only if there are $\delta, \varepsilon$ in $(0,1)$ such that $|g(z)| \geq \varepsilon$ for all $\delta \leq|z|<1$. By the symbol calculus

$$
\mathrm{BCESV} / C_{0}(\mathbf{D}) \cong \tau(\mathrm{BCESV}) / \mathcal{K}
$$

$T_{g}+\mathcal{K}$ is invertible in $\tau$ (BCESV) $/ \mathcal{K}$ if and only if there are $\delta, \varepsilon$ in $(0,1)$ such that $|g(z)| \geq \varepsilon$ for all $\delta \leq|z|<1$. Therefore

$$
\sigma_{e}\left(T_{\tilde{f}}\right)=\bigcap_{\delta \in(0,1)} \overline{\tilde{f}\left(\mathbf{D}-\mathbf{D}_{\delta}\right)},
$$

where $\mathbf{D}_{\delta}=\{z \in \mathbf{D}|| z \mid<\delta\}$. The compactness of $\beta \mathbf{D}$ and the continuity of $\tilde{f}$ yield $\tilde{f} \overline{\left(\mathbf{D}-\mathbf{D}_{\delta}\right)}=\tilde{f} \overline{\left(\mathbf{D}-\mathbf{D}_{\delta}\right)}=\tilde{f}\left(\beta \mathbf{D}-\mathbf{D}_{\delta}\right)$. So we get

$$
\sigma_{e}\left(T_{\tilde{f}}\right)=\bigcap_{\delta \in(0,1)} \tilde{f}\left(\beta \mathbf{D}-\mathbf{D}_{\delta}\right) .
$$

On the other hand, if $\lambda \in \bigcap_{\delta \in(0,1)} \tilde{f}\left(\beta \mathbf{D}-\mathbf{D}_{\delta}\right)$, then $\lambda=\tilde{f}\left(z_{\delta}\right), z_{\delta} \in \beta \mathbf{D}-\mathbf{D}_{\delta}, \delta \in$ $(0,1)$. Consider the sequence $\left\{z_{1-1 / n}\right\}$. The compactness of $\beta \mathrm{D}$ implies that there exists a subsequence $\left\{z_{1-1 / n_{k}}\right\}$ and $z \in \beta \mathrm{D}$ such that $z_{1-1 / n_{k}} \rightarrow z$ as $k \rightarrow+\infty$. It is clear that $z \in \beta \mathbf{D}-\mathbf{D}$ since $\mathbf{D}$ is open in $\beta \mathbf{D}$. The continuity of $\tilde{f}$ and the equality $\lambda=f\left(z_{\delta}\right)$ give $\lambda=\tilde{f}(z) \in \tilde{f}(\beta \mathbf{D}-\mathbf{D})$. Hence $\tilde{f}(\beta \mathbf{D}-\mathbf{D})=\bigcap_{\delta \in(0,1)} \tilde{f}\left(\beta \mathbf{D}-\mathbf{D}_{\delta}\right)$, and the proof is complete.

COROLlaRY 1. For $f \in Q, T_{f}$ is Fredholm if and only if $\tilde{f}$ is nonvanishing on $\{z|z \in \mathbf{D}| z \mid, \geq \delta\}$ for some $\delta \in(0,1)$.

COROllaRY 2. If $f \in \mathrm{BCESV}$, then $\sigma_{e}\left(T_{f}\right)=f(\beta \mathbf{D}-\mathbf{D})$, hence $T_{f}$ is Fredholm if and only if $f$ is nonvanishing on $\{z|z \in \mathbf{D}| z \mid, \geq \delta\}$ for some $\delta \in(0,1)$.

Proof. For $f \in \mathrm{BCESV}, f-\tilde{f}$ is in $C_{0}(\mathbf{D})$, so $f(\beta \mathbf{D}-\mathbf{D})=\tilde{f}(\beta \mathbf{D}-\mathbf{D})$.

COROLlaRY 3. If $f \in Q$, then $\sigma_{e}\left(T_{f}\right)$ is connected.

ProOF. $\sigma_{e}\left(T_{f}\right)=\bigcap_{\delta \in(0,1)} \overline{\tilde{f}\left(\mathbf{D}-\mathbf{D}_{\delta}\right)}$ is the intersection of a nested family of compact connected sets, so it is connected. See [7]. 
REMARK. As $C^{*}$-algebras, $C(\beta \mathbf{D})$ is isomorphic to $\mathrm{BC}(\mathbf{D})$. Under the isomorphism, $C_{0}(\mathbf{D})$ is the closed ideal of $C(\beta \mathbf{D})$ consisting of functions $f$ on $\beta \mathbf{D}$ such that $f$ is identically zero on $\beta \mathbf{D}-\mathbf{D}$.

If $f$ is in BCESV and $T_{f}$ is Fredholm, then we know that there are $\delta, \varepsilon \in(0,1)$ such that $|f(z)| \geq \varepsilon$ for all $\delta \leq|z|<1$. For any $r \in(\delta, 1)$, we have a continuous map $f_{r}: \partial \mathbf{D} \rightarrow C-\{0\}$ defined by $f_{r}\left(e^{i \theta}\right)=f\left(r e^{i \theta}\right)$. Given any two $r_{1}, r_{2} \in[\delta, 1), f_{r_{1}}$ and $f_{r_{2}}$ are homotopic in the obvious way. So the winding numbers of $f_{r_{1}}$ and $f_{r_{2}}$ are equal and independent of the choice of $\delta$. Denote the common winding number by $\mathcal{N}_{f}$. Then by monodromy as used in [7], we can prove

THEOREM 15. If $f \in Q$ and $T_{f}$ is Fredholm, then $\operatorname{Ind}\left(T_{f}\right)=-\mathcal{N}_{\tilde{f}}$, where $\operatorname{Ind}\left(T_{f}\right)$ is the Fredholm index of $T_{f}$, i.e. $\operatorname{Ind}\left(T_{f}\right)=$ dimension of kernel $T_{f}-$ dimension of kernel $T_{\bar{f}}$.

REMARK. BCESV has played a significant role in our analysis. It seems interesting to know the structure of BCESV as a $C^{*}$-algebra. BCESV contains $C(\overline{\mathbf{D}})$ as a proper $C^{*}$-subalgebra. Let $\mathcal{M}$ be the maximal ideal space of BCESV. $\mathcal{M}$ is connected since for any $f \in \mathrm{BCESV}, \sigma(f)=\overline{f(\mathbf{D})}$ is connected (so there is no idempotent in BCESV with spectrum $\{0,1\})$. For any $\lambda \in \mathbf{D}$, the evaluation functional on BCESV at $\lambda$ is in $\mathcal{M}$, denoted by $F_{\lambda}$. The map $\lambda \mapsto F_{\lambda}$ is a one-to-one map of $\mathbf{D}$ into $\mathcal{M}$. Let $D$ be the image of this map. We put the induced topology on $D$. Let $f_{0} \in \mathrm{BCESV}$ be the function $f_{0}(z)=z$ for all $z \in \mathbf{D}$. Then we have the following

THEOREM 16. Let $F \in \mathcal{M}$ be a multiplicative linear functional on BCESV. Then $F \in D$ if and only if $\left|F\left(f_{0}\right)\right|<1$.

ProOF. The "only if" part is obvious. We prove the "if" part.

Suppose $\left|F\left(f_{0}\right)\right|<1$. Let $z_{0}=F\left(f_{0}\right) \in \mathbf{D}$. We want to prove $F(f)=f\left(z_{0}\right)$ for all $f$ in BCESV. By the Stone-Weierstrass approximation theorem, it is easy to show that $F(f)=f\left(z_{0}\right)$ for all $f$ in $C(\overline{\mathbf{D}})$. Choose a function $\varphi \in(\overline{\mathbf{D}})$ so that $\varphi \equiv 1$ on a neighborhood $U \subset \mathbf{D}$ of $z_{0}$ and $\varphi \equiv 0$ on a neighborhood $V$ of $\partial \mathbf{D}$. Now for any $f \in \mathrm{BCESV}, f \varphi \in C(\overline{\mathbf{D}})$. Thus $F(f \varphi)=(f \varphi)\left(z_{0}\right)=f\left(z_{0}\right) \varphi\left(z_{0}\right)$. On the other hand, the multiplicativity of $F$ given $F(f \varphi)=F(f) F(\varphi)=F(f) \varphi\left(z_{0}\right)=F(f)$. Hence $F(f)=f\left(z_{0}\right)$ for all $f \in \mathrm{BCESV}$.

COROLlary. $D$ is open in $\mathcal{M}$, and hence $\mathrm{BCESV} / C_{0}(\mathbf{D}) \cong C(\mathcal{M}-D)$.

Proof. The map $F \mapsto F\left(f_{0}\right)$ from $\mathcal{M}$ to $C$ is continuous. By the above theorem, $D$ is the inverse image of $\mathbf{D}$ under this map, so $D$ is open in $\mathcal{M}$.

REMARK. This corollary says that $\mathcal{M}-D$ is homeomorphic to the maximal ideal space of $\mathrm{BCESV} / C_{0}(\mathbf{D}) \cong \tau(Q) / K$.

REMARK. $\mathcal{M}-D$ is connected since $\mathrm{BCESV} / C_{0}(\mathbf{D})$ has no idempotent element with spectrum $\{0,1\}$ by Corollary 3 to Theorem 14 .

7. A conformal invariant description of $\mathrm{VMO}_{\partial}$. In this section, we are going to give another characterization of $\mathrm{VMO}_{\partial}$. Also we will describe the relationship between $\mathrm{VMO}_{\partial}(\mathbf{D})$ and the usual $\operatorname{VMO}(\mathbf{D})$.

For $z_{0} \in \mathbf{D}$ and $r \in(0,1)$, let

$$
D\left(z_{0}, r\right)=\left\{z \in \mathbf{D}:\left|\left(z_{0}-z\right) /\left(1-\bar{z}_{0} z\right)\right|<r\right\} .
$$


$D\left(z_{0}, r\right)$ is called the pseudohyperbolic disc centered at $z_{0}$ with radius $r$. It is actually a Euclidean disc (see $[\mathbf{1 3}]$ ) contained in $\mathbf{D}$ with center

$$
c=\frac{1-r^{2}}{1-r^{2}\left|z_{0}\right|^{2}} z_{0}
$$

and radius

$$
R=r \frac{1-\left|z_{0}\right|^{2}}{1-r^{2}\left|z_{0}\right|^{2}}
$$

Thus the normalized Lebesgue measure of $D\left(z_{0}, r\right)$ is

$$
\left|D\left(z_{0}, r\right)\right|=r^{2}\left(1-\left|z_{0}\right|^{2}\right)^{2} /\left(1-r^{2}\left|z_{0}\right|^{2}\right)^{2} .
$$

THEOREM 17. For $f \in L^{\infty}(\mathbf{D}, d A)$, we have $f \in \mathrm{VMO}_{\partial}$ if and only if

$$
\lim _{|z| \rightarrow 1} \frac{1}{|D(z, r)|} \int_{D(z, r)}\left|f(w)-\frac{1}{|D(z, r)|} \int_{D(z, r)} f(u) d A(u)\right| d A(w)=0
$$

for each $r \in(0,1)$.

ProOF. Let

$$
I(z, r)=\frac{1}{|D(z, r)|^{2}} \int_{D(z, r)} \int_{D(z, r)}|f(u)-f(w)|^{2} d A(w) d A(u) .
$$

Since $f$ is bounded, it suffices to show that $f \in \mathrm{VMO}_{\partial} \Leftrightarrow I(z, r) \rightarrow 0$ as $|z| \rightarrow 1$ for each $r \in(0,1)$.

A change of variable shows that

$$
\begin{aligned}
I(z, r) & =\frac{1}{|D(z, r)|^{2}} \int_{|w| \leq r} \int_{|u| \leq r}\left|f\left(\frac{z-w}{1-\bar{z} w}\right)-f\left(\frac{z-u}{1-\bar{z} u}\right)\right|^{2} \\
& \leq \frac{1}{|D(z, r)|^{2}} \frac{\left(1-|z|^{2}\right)^{4}}{(1-r)^{8}} \int_{\mathbf{D}} \int_{\mathbf{D}}\left|f\left(\frac{z-w}{1-\bar{z} w}\right)-f\left(\frac{z-u}{1-\bar{z} u}\right)\right|^{2} d A(w) d A(u) \\
& =\frac{2\left(1-r^{2}|z|^{2}\right)^{4}}{r^{4}(1-r)^{8}}\left(\widetilde{|f|^{2}}(z)-|\tilde{f}(z)|^{2}\right) \\
& \leq \frac{2}{r^{4}(1-r)^{8}}\left(\widetilde{|f|^{2}}(z)-|\tilde{f}(z)|^{2}\right) .
\end{aligned}
$$

Thus $f \in \mathrm{VMO}_{\partial} \Rightarrow f \in \tilde{Q} \Rightarrow I(z, r) \rightarrow 0$ as $|z| \rightarrow 1$ for each $r \in(0,1)$.

On the other hand,

$$
\begin{aligned}
I(z, r) & \geq \frac{\left(1-|z|^{2}\right)^{4}}{2^{8}|D(z, r)|^{2}} \int_{|w| \leq r} \int_{|u| \leq r}\left|f\left(\frac{z-w}{1-\bar{z} w}\right)-f\left(\frac{z-u}{1-\bar{z} u}\right)\right|^{2} d A(w) d A(u) \\
& =\frac{\left(1-r^{2}|z|^{2}\right)^{4}}{2^{8} r^{4}}\left[\int_{\mathbf{D}} \int_{\mathbf{D}}-\int_{\mathbf{D}} \int_{\mathbf{D}-\mathbf{D}_{r}}-\int_{\mathbf{D}-\mathbf{D}_{r}} \int_{\mathbf{D}_{r}}\right] \\
& \geq \frac{\left(1-r^{2}|z|^{2}\right)^{4}}{2^{8} r^{4}}\left[\int_{\mathbf{D}} \int_{\mathbf{D}}-8\|f\|_{\infty}^{2}\left|\mathbf{D}-\mathbf{D}_{r}\right|\right]
\end{aligned}
$$


that is,

$$
2\left(\widetilde{|f|^{2}}(z)-|\tilde{f}(z)|^{2}\right) \leq \frac{2^{8} r^{4}}{\left(1-r^{2}|z|^{2}\right)^{4}} I(z, r)+8\|f\|_{\infty}^{2}\left|\mathbf{D}-\mathbf{D}_{r}\right| .
$$

Now if $I(z, r) \rightarrow 0$ as $|z| \rightarrow 1$ for each $r \in(0,1)$, then

$$
2 \varlimsup_{|z| \rightarrow 1}\left(\widetilde{\left.f\right|^{2}}(z)-|\tilde{f}(z)|^{2}\right) \leq 8\|f\|_{\infty}^{2}\left|\mathbf{D}-\mathbf{D}_{r}\right|
$$

for each $r \in(0,1)$. Letting $r \rightarrow 1$ yields

$$
\lim _{|z| \rightarrow 1}\left(\widetilde{|f|^{2}}(z)-|\tilde{f}(z)|^{2}\right)=0
$$

namely, $f \in \tilde{Q}=\mathrm{VMO}_{\partial} \cap L^{\infty}$.

COROLlaRY 1. For $f \in L^{\infty}(\mathbf{D}, d A)$, we have $f \in Q \cap B$ if and only if

$$
\lim _{|z| \rightarrow 1} \frac{1}{|D(z, r)|} \int_{D(z, r)}|f(w)| d A(w)=0
$$

for each $r \in(0,1)$.

The proof of Corollary 1 is very similar to that of the theorem, so we omit it.

For any $f \in L^{\infty}(\mathbf{D}, d A)$, define a continuous function $\hat{f}_{r}(z)$ on $\mathbf{D}$ as follows:

$$
\hat{f}_{r}(z)=\frac{1}{|D(z, r)|} \int_{D(z, r)} f(u) d A(u) .
$$

Then we have

Corollary 2. For $f \in L^{\infty}(\mathbf{D}, d A)$, we have

$$
\begin{aligned}
& f \in Q \Leftrightarrow \widehat{|f|_{r}^{2}}(z)-\left|\hat{f}_{r}(z)\right|^{2} \rightarrow 0(|z| \rightarrow 1) \quad \text { for any } r \in(0,1), \\
& f \in Q \cap B \Leftrightarrow \widehat{|f|_{r}}(z) \rightarrow 0(|z| \rightarrow 1) \quad \text { for any } r \in(0,1) .
\end{aligned}
$$

ProOF. The second equivalence is just the above Corollary 1. The first equivalence follows from the identity

$$
I(z, r)=2\left(\widehat{|f|_{r}^{2}}(z)-\left|\hat{f}_{r}(z)\right|^{2}\right) .
$$

THEOREM 18. For $f \in L^{\infty}(\mathbf{D}, d A)$, we have

$$
\begin{aligned}
& f \in Q \Leftrightarrow \lim _{|z| \rightarrow 1} \frac{1}{|D(z, r)|} \int_{D(z, r)}|f(w)-\tilde{f}(z)|^{2} d A(w)=0, \\
& f \in \mathrm{ESV} \Leftrightarrow \lim _{|z| \rightarrow 1} \frac{1}{|D(z, r)|} \int_{D(z, r)}|f(w)-f(z)|^{2} d A(w)=0 .
\end{aligned}
$$

ProOF. Recall that

$$
\begin{aligned}
& f \in Q \Leftrightarrow\left\|f \circ b_{z}-\tilde{f}(z)\right\|_{L^{2}} \rightarrow 0 \quad \text { as }|z| \rightarrow 1, \\
& f \in \mathrm{ESV} \Leftrightarrow\left\|f \circ b_{z}-f(z)\right\|_{L^{2}} \rightarrow 0 \quad \text { as }|z| \rightarrow 1 .
\end{aligned}
$$

Now the theorem can be proved by using the same techniques as in the proof of Theorem 17. 
COROllary 1. For $f \in Q$, we have

$$
f \in \mathrm{ESV} \Leftrightarrow \hat{f}_{r}(z)-f(z) \rightarrow 0 \quad \text { as }|z| \rightarrow 1 \text { for each } r \in(0,1) .
$$

ProOF. The proof follows from Corollary 2 to Theorem 17 and the identity

$$
\frac{1}{|D(z, r)|} \int_{D(z, r)}|f(w)-f(z)|^{2} d A(w)=\widehat{|f|_{r}^{2}}(z)-\left|\hat{f}_{r}(z)\right|^{2}+\left|\hat{f}_{r}(z)-f(z)\right|^{2}
$$

COROllary 2. For $f \in Q$, we have

$$
\hat{f}_{r}(z)-\tilde{f}(z) \rightarrow 0 \quad \text { as } \quad|z| \rightarrow 1 \text { for each } r \in(0,1) .
$$

PROOF.

$$
\left|\hat{f}_{r}(z)-\tilde{f}(z)\right| \leq \frac{1}{|D(z, r)|} \int_{D(z, r)}|f(w)-\tilde{f}(z)| d A(w) .
$$

Now the assertion follows from Theorem 18 and the Schwarz inequality.

COROLlaRY 3. For $f \in Q$, we have $\hat{f}_{r} \in \mathrm{ESV}$ and $f-\hat{f}_{r} \in Q \cap B$.

PROOF. It follows from Corollary 2 and the fact that $\tilde{f} \in \operatorname{ESV}$ and $f-\tilde{f} \in Q \cap B$.

COROllary 4. Given $f \in L^{\infty}(\mathbf{D})$, we have

$$
f \in \mathrm{ESV} \Leftrightarrow \lim _{|z| \rightarrow 1^{-}} \sup _{w \in D(z, r)}|f(z)-f(w)|=0
$$

for all $r \in(0,1)$.

PROOF. " $\Leftarrow$ " follows from the second statement of the theorem.

To prove " $\Rightarrow$ ", given any $r \in\left(0, \frac{1}{2}\right)$ and consider $\hat{f}_{r}(z)$ on D. Suppose $w \in$ $D(z, r)$. Then

$$
\begin{aligned}
\mid \hat{f}_{r}(z) & -\hat{f}_{r}(w)\left|\leq \frac{1}{|D(z, r)||D(w, r)|} \int_{D(z, r)} \int_{D(w, r)}\right| f(u)-f(v) \mid d A(u) d A(v) \\
& \leq \frac{|D(z, 2 r)|^{2}}{|D(z, r)||D(w, r)|} \frac{1}{|D(z, 2 r)|^{2}} \cdot \int_{D(z, 2 r)} \int_{D(z, 2 r)}|f(u)-f(v)| d A(u) d A(v)
\end{aligned}
$$

Since $f \in \mathrm{ESV} \Rightarrow f \in \mathrm{VMO}_{\partial}(\mathbf{D})$, we have

$$
\lim _{|z| \rightarrow 1^{-}} \sup _{w \in D(z, r)}\left|\hat{f}_{r}(z)-\hat{f}_{r}(w)\right|=0 \quad\left(r \in\left(0, \frac{1}{2}\right)\right),
$$

but $f(z)-\hat{f}_{r}(z) \rightarrow 0$ as $|z| \rightarrow 1^{-}$, hence

$$
\lim _{|z| \rightarrow 1^{-}} \sup _{w \in D(z, r)}|f(z)-f(w)|=0 \quad\left(r \in\left(0, \frac{1}{2}\right)\right) .
$$

By a finite covering argument, we get

$$
\lim _{|z| \rightarrow 1^{-}} \sup _{w \in D(z, r)}|f(z)-f(w)|=0
$$

for all $r \in(0,1)$.

Finally, we discuss the relationship between $\mathrm{VMO}_{\partial}(\mathbf{D})$ and the usual area $\operatorname{VMO}(\mathbf{D})$. Recall that $f \in \operatorname{VMO}(\mathbf{D})$ if and only if given $\varepsilon>0$, there is $\delta \in(0,1)$ such that

$$
\frac{1}{|D|} \int_{D}\left|f(w)-\frac{1}{|D|} \int_{D} f(u) d A(u)\right| d A(w)<\varepsilon
$$


whenever $D$ is a disc contained in $\mathbf{D}$ with radius $\leq \delta$. For any $r \in(0,1)$, the pseudohyperbolic disc $D(z, r)$ centered at $z$ is a Euclidean disc contained in $\mathbf{D}$ with radius

$$
R=r \frac{1-|z|^{2}}{1-r^{2}|z|^{2}}
$$

which goes to 0 as $|z| \rightarrow 1$. Thus if $f \in \operatorname{VMO}(\mathbf{D})$, then for any $r \in(0,1)$ we have

$$
\lim _{|z| \rightarrow 1} \frac{1}{|D(z, r)|} \int_{D(z, r)}\left|f(w)-\frac{1}{|D(z, r)|} \int_{D(z, r)} f(u) d A(u)\right| d A(w)=0 .
$$

THEOREM 19. If $f \in L^{\infty}(\mathbf{D})$, then $f \in \mathrm{VMO}(\mathbf{D}) \Rightarrow f \in \mathrm{VMO}_{\partial}(\mathbf{D})$.

REMARK. The converse of Theorem 19 is obviously false. For example, if $f$ is the characteristic function of any closed square contained in $\mathbf{D}$, then $f \in Q \cap B \subset$ $\mathrm{VMO}_{\partial}(\mathbf{D})$ while $f \notin \mathrm{VMO}(\mathbf{D})$. Even for bounded continuous functions $f$ on $\mathbf{D}$, the converse of Theorem 19 does not hold. However, if $f \in H^{\infty}(\mathbf{D})$, then $f \in \mathrm{VMO}_{\partial}(\mathbf{D}) \Leftrightarrow f \in \mathrm{VMO}(\mathbf{D})$.

8. Open questions and possible generalizations. All the results in this paper are concerned with essentially bounded functions on $\mathbf{D}$. It is clear that many concepts and techniques apply to unbounded functions. First we make some definitions.

Definition 1. A function $f \in L^{1}(\mathbf{D})$ is said to be in $\mathrm{BMO}_{\partial}(\mathbf{D})$ if

$$
\sup _{z \in \mathbf{D}} \frac{1}{\left|S_{z}\right|} \int_{S_{z}}\left|f(w)-\frac{1}{\left|S_{z}\right|} \int_{S_{z}} f(u) d A(u)\right| d A(w)<+\infty .
$$

It is obvious that $\mathrm{VMO}_{\partial}(\mathbf{D}) \subset \mathrm{BMO}_{\partial}(\mathbf{D})$.

For a function $f \in L^{2}(\mathbf{D}, d A)$, the Toeplitz operator $T_{f}$ is an unbounded operator in general. However, we always have $k_{z} \in D\left(T_{f}\right)$. Thus, the Berezin symbol $\tilde{f}$ is well defined in this case. Also $\hat{f}$ is well defined. Our first problem is to generalize Theorem 1:

Problem 1. For $f \in L^{2}(\mathbf{D}, d A)$, prove that the following are all equivalent:

(a) $H_{f}$ and $H_{\vec{f}}$ are compact;

(b) $T_{|f|^{2}}-T_{f} T_{\bar{f}}$ and $T_{|f|^{2}}-T_{\bar{f}} T_{f}$ are compact;

(c) $f \in \mathrm{VMO}_{\partial}(\mathbf{D})$;

(d) $\widetilde{|f|^{2}}(z)-|\tilde{f}(z)|^{2} \rightarrow 0$ as $|z| \rightarrow 1$;

(e) $\widetilde{|f|^{2}}(z)-|\hat{f}(z)|^{2} \rightarrow 0$ as $|z| \rightarrow 1$.

An analogous problem is

Problem 2. For $f \in L^{2}(\mathbf{D}, d A)$, prove that the following are all equivalent:

(a) $H_{f}$ and $H_{\vec{f}}$ are bounded;

(b) $T_{|f|^{2}}-T_{f} T_{\bar{f}}$ and $T_{|f|^{2}}-T_{\bar{f}} T_{f}$ are bounded;

(c) $f \in \mathrm{BMO}_{\partial}(\mathbf{D})$;

(d) $\widetilde{|f|^{2}}(z)-|\tilde{f}(z)|^{2}$ is bounded on $\mathbf{D}$;

(e) $\widehat{|f|^{2}}(z)-|\hat{f}(z)|^{2}$ is bounded on $\mathbf{D}$. 
For $f \in L^{2}(\mathbf{D}, d A)$, let

$$
\begin{aligned}
\|f\|_{1} & =\sup _{z \in \mathrm{D}} \frac{1}{\left|S_{z}\right|} \int_{S_{z}}\left|f(w)-\frac{1}{\left|S_{z}\right|} \int_{S_{z}} f(u) d A(u)\right| d A(w), \\
\|f\|_{2} & =\sup _{z \in \mathbf{D}} \frac{1}{\left|S_{z}\right|} \sqrt{\int_{S_{z}} \int_{S_{z}}|f(u)-f(w)|^{2} d A(u) d A(w)} \\
\|f\|_{3} & =\sup _{z \in \mathrm{D}} \sqrt{\left.\left|\widetilde{\left.f\right|^{2}}(z)-\right| \tilde{f}(z)\right|^{2}} .
\end{aligned}
$$

Problem 3. Show that \|\|$_{i}(i=1,2,3)$ are complete norms on $\mathrm{BMO}_{\partial}(\mathbf{D})$ modula the constant functions and show that they are equivalent.

In the theory of BMO and VMO [13], Fefferman's duality theorem is one of the most important and deepest results, so it is very natural to propose:

Problem 4. Formulate and prove a duality theorem about $\mathrm{BMO}_{\partial}(\mathbf{D})$.

New characterizations of $\mathrm{BMO}_{\partial}(\mathbf{D})$ and $\mathrm{VMO}_{\partial}(\mathbf{D})$ are also worth further investigation.

Finally, I am very curious about the possible generalizations of the above concepts and results to general strongly pseudo-convex domains $\Omega$ in $\mathbf{C}^{n}$. The definitions of Berezin symbol, $Q$, and $\tilde{Q}$ can be carried over word by word. It seems to me that a reasonable definition of $\operatorname{BMO}_{\partial}(\Omega)$ and $\operatorname{VMO}_{\partial}(\Omega)$ as well as $\operatorname{ESV}(\Omega)$ should involve the geometry of $\Omega$ and $\partial \Omega$. A connection between geometry and operator theory is expected in the further study of this direction.

ACKNOWLEDGMENTS. This paper is my Ph.D. thesis, written at the State University of New York at Buffalo under the supervision of Professor Lewis A. Coburn. I am grateful to Professor Donald Sarason for helpful conversations and for inviting me to talk about this work at his analysis seminar in Berkeley. Special thanks go to Professor Sheldon Axler for useful conversations and for giving me access to the manuscript of his forthcoming paper, "The Bergman space, the Bloch space, and commutators of multiplication operators". Professor Charles A. Berger helped me greatly with some of his unpublished results and ideas.

\section{REFERENCES}

1. Sheldon Axler, The Bergman space, the Bloch space, and commutators of multiplication operators, Duke Math. J. 53 (1986) 315-332.

2. Sheldon Axler, John Conway, and G. MacDonald, Toeplitz operators on the Bergman spaces, Canad. J. Math. 34 (1982), 466-483.

3. Sheldon Axler, Hankel operators on Bergman spaces, Linear and Complex Analysis Problem Book (V. P. Havin, S. V. Hrušs̈ë, and N. K. Nikol'skii, eds.), Lecture Notes in Math., Vol. 1043, Springer-Verlag, Berlin, 1984, pp. 262-263.

4. V. Bargmann, Remarks on a Hilbert space of analytic functions, Proc. Nat. Acad. Sci. U.S.A. 48 (1962), 199-204.

5. F. A. Berezin, Covariant and contravariant symbols of operators, Math. USSR-Izv. 6 (1972), 1117-1151.

6. C. A. Berger and L. A. Coburn, Toeplitz operators and quantum mechanics, J. Funct. Anal. 68 (1986), 273-299.

7. 813-829.

8. S. Bergman, The kernel functions and conformal mapping, Math. Surveys, No. 5, Amer. Math. Soc., Providence, R.I., 1950. 
9. L. A. Coburn, Singular integral operators and Toeplitz operators on odd spheres, Indiana Univ. Math. J. 23 (1973), 433-439.

10. R. R. Coifman, R. Rochberg, and G. Weiss, Factorization for Hardy spaces, Ann. of Math. 103 (1976), 611-635.

11. R. Douglas, Banach algebra techniques in operator theory, Academic Press, New York, 1972.

12. P. L. Duren, Theory of $H^{p}$ spaces, Academic Press, New York, 1970.

13. John B. Garnett, Bounded analytic functions, Academic Press, New York, 1981.

14. V. Guillemin, Toeplitz operators in n-dimensions, Integral Equations and Operator Theory, Vol. 7, 1984, pp. 145-205.

15. P. Hartman, On completely continuous Hankel matrices, Proc. Amer. Math. Soc. 9 (1958), 862-866.

16. Nicholas P. Jewell, Toeplitz operators on the Bergman space and in several complex variables, Proc. London Math. Soc. 41 (1980), 193-216.

17. U. Venugopolkrishna, Fredholm operators associated with strongly pseudo-convex domains in $\mathbf{C}^{n}$, J. Funct. Anal. 9 (1972), 349-373.

18. Steven G. Krantz, Function theory of several complex variables, Wiley, New York, 1982.

19. Gerard McDonald, Fredholm properties of a class of Toeplitz operators on the ball, Indiana Univ. Math. J. 26 (1977), 567-576.

20. Gerard McDonald and C. Sundberg, Toeplitz operators on the disc, Indiana Univ. Math. J. 28 (1979), 595-611.

21. Walter Rudin, Function theory in the unit ball of $\mathbf{C}^{n}$, Springer, Berlin, 1980.

22. Donald Sarason, Functions of vanishing mean oscillation, Trans. Amer. Math. Soc. 207 (1975), 391-405.

23. _ _ Toeplitz operators with piecewise quasicontinuous symbols, Indiana Univ. Math. J. 26 (1977).

Department of Mathematics, University of Washington, Seattle, WashingTON 98195 